\title{
The spinor and tensor fields with higher spin on spaces of constant curvature
}

\author{
Yasushi Homma ${ }^{1}$ Takuma Tomihisa ${ }^{2}$
}

Received: 7 September 2020 / Accepted: 4 July 2021 / Published online: 26 July 2021

(C) The Author(s) 2021

\begin{abstract}
In this article, we give all the Weitzenböck-type formulas among the geometric first-order differential operators on the spinor fields with spin $j+1 / 2$ over Riemannian spin manifolds of constant curvature. Then, we find an explicit factorization formula of the Laplace operator raised to the power $j+1$ and understand how the spinor fields with spin $j+1 / 2$ are related to the spinors with lower spin. As an application, we calculate the spectra of the operators on the standard sphere and clarify the relation among the spinors from the viewpoint of representation theory. Next we study the case of trace-free symmetric tensor fields with an application to Killing tensor fields. Lastly we discuss the spinor fields coupled with differential forms and give a kind of Hodge-de Rham decomposition on spaces of constant curvature.
\end{abstract}

Keywords The Lichnerowicz Laplacian · The (higher spin) Dirac · Weitzenböck formulas · Generalized gradients $\cdot$ Space of constant curvature $\cdot$ Harmonic analysis on spheres

Mathematics Subject Classification $53 \mathrm{C} 27 \cdot 58 \mathrm{C} 40 \cdot 58 \mathrm{~J} 50$

\section{Introduction}

Recently, many researchers in mathematics and physics have tried to understand geometry and analysis for the spinor fields with higher spin and the symmetric tensor fields. In fact, there are a lot of articles for the Rarita-Schwinger fields with spin $3 / 2$ and Killing tensor fields, which are special fields satisfying geometric differential equations. One of the methods to define such meaningful geometric differential equations is to use the generalized gradients or the Stein-Weiss operators. The operators are first-order differential operators

Takuma Tomihisa

taku-tomihisa@akane.waseda.jp

Yasushi Homma

homma_yasushi@waseda.jp

1 Department of Mathematics, Faculty of science and engineering, Waseda University, 3-4-1

Ohkubo, Shinjuku-ku, Tokyo 169-8555, Japan

2 Department of Applied Mathematics, Faculty of science and engineering, Waseda University, 3-4-1 Ohkubo, Shinjuku-ku, Tokyo 169-8555, Japan 
naturally defined on spinor and tensor fields. In [12], Fegan classified them and showed they are conformally covariant. In [4], Branson studied the ellipticity and Weitzenböck formulas for generalized gradients using spectra of them on the standard sphere. He also studied another type of Weitzenböck formula in [5]. After a decade, the first author of this paper showed an explicit method to construct the Weitzenböck formulas for the generalized gradients in [16], which produce a lot of applications, vanishing theorem, eigenvalue estimates and so on. There are also many articles to study the generalized gradients and their applications to mathematics and physics ([15, 17, 18, 22], etc.). Moving on to analysis, we know that one of the main topics in Clifford analysis is to generalize spherical harmonic analysis on Euclidean space to such spinor and tensor fields. Polynomial solutions called monogenic functions, fundamental solutions and Fueter theorem for the higher spin Dirac operators have been constructed in [9-11], etc. The key to give the fundamental solution is a so-called factorization formula. The $j+1$-st power of the Laplace operator $\Delta$ on $\mathbb{R}^{n}$ is factorized as $\Delta^{j+1}=D_{j} \circ A_{2 j+1}$, where $D_{j}$ is a generalized gradient (the higher spin Dirac operator) and $A_{2 j+1}$ is a differential operator with order $2 j+1$. To consider such a formula on curved manifold would be important to analyze higher spin fields and give a variety of applications to geometry.

In this article, we combine Clifford analysis and differential geometry. We study the generalized gradients on spinor fields with spin $j+1 / 2$ over a Riemannian spin manifold $(M, g)$ and try to give a factorization formula for the exponentiation of the Laplace operator $\Delta$. Since Weitzenböck formulas are too complicated for $j \geq 2$ to handle them on curved manifolds, we assume that $(M, g)$ has a constant sectional curvature. Then, we see any operators commute with each other in a way and construct a factorization formula explicitly in Theorem 2.9. To clarify the meaning of the factorization, we show how the spinor fields with spin $j+1 / 2$ are influenced by the spinor fields with lower spin in Theorem 2.11. Remark that, in the case of $j=1$, we need only the assumption of Einstein manifold and can develop fruitful geometry and analysis in [1, 18] and [19]. Next, we study harmonic analysis on spinor fields with spin $j+1 / 2$ on the standard sphere as a model case for spinor analysis on a curved space in Sect. 3. Interestingly the factorization formula and Weitzenböck formulas yield all the eigenvalues of the generalized gradients from the eigenvalues of the Laplacian, which are calculated by Freudenthal's formula for Casimir operator (cf. [3]). This method would be easier to understand than in [6] or originally in [7]. We also show how the spaces of the spinor fields as Spin-modules relate to each other through the generalized gradients. In Sect. 4, we discuss the trace-free symmetric tensor fields on a space of constant curvature and give a factorization formula in Theorem 4.5. We study harmonic analysis on such fields over the standard sphere in Sect. 5. As an application, we give a decomposition of the Killing tensor fields on the sphere from the viewpoint of representation theory. In Sects. 6 and 7, we discuss the spinor fields coupled with differential forms on a space of constant curvature and harmonic analysis on the sphere. In particular, we give a kind of Hodge-de Rham decomposition for spinor fields with differential forms. In Appendix A, we show how to calculate the Weitzenböck formulas needed in Sect. 2.

\section{Higher spin Dirac operators on spinor fields with spin $j+1 / 2$}

Let $(M, g)$ be an $n$-dimensional Riemannian spin manifold with a spin structure $\operatorname{Spin}(M)$, which is a principal $\operatorname{Spin}(n)$-bundle over $M$ and doubly covering the orthonormal frame bundle $\mathrm{SO}(M)$. Throughout this paper, we assume $n \geq 3$. The case of dimension two is 
left as an exercise to the reader. We consider the spin $j+1 / 2$ (unitary) representation $\pi_{j}$ on $W_{j}$ of $\operatorname{Spin}(n)$ for $j=0,1, \cdots$. For example, $\left(\pi_{0}, W_{0}\right)$ is a usual spinor representation. For $n=2 m$, the space $W_{j}$ is decomposed into the direct sum of $W_{j}^{+}$and $W_{j}^{-}$. Each $W_{j}^{ \pm}$is an irreducible $\operatorname{Spin}(n)$-module whose highest weight is

$$
(j+1 / 2, \underbrace{1 / 2, \cdots, 1 / 2}_{m-2}, \pm 1 / 2)=\left(j+1 / 2,(1 / 2)_{m-2}, \pm 1 / 2\right) .
$$

Here, $(1 / 2)_{k}$ denotes a sequence $1 / 2, \cdots, 1 / 2$ with length $k$ as an abbreviation. We write the direct sum representation $\pi_{j}^{+} \oplus \pi_{j}^{-}$by $\pi_{j}$. For $n=2 m-1$, the spin $j+1 / 2$ spinor space $W_{j}$ is an irreducible $\operatorname{Spin}(n)$-module whose highest weight is $\left(j+1 / 2,(1 / 2)_{m-2}\right)$. It follows from Weyl's dimension formula that the dimension of $W_{j}$ is

$$
\operatorname{dim} W_{j}=2^{[n / 2]}\left(\begin{array}{c}
n+j-2 \\
j
\end{array}\right), \quad \operatorname{dim} W_{j}^{ \pm}=2^{[n / 2]-1}\left(\begin{array}{c}
n+j-2 \\
j
\end{array}\right) .
$$

The representation $\pi_{j}$ (resp. $\pi_{j}^{ \pm}$) induces a vector bundle $S_{j}$ (resp. $S_{j}^{ \pm}$) associated with the principal bundle $\operatorname{Spin}(M)$. Indeed, we consider the action of $\operatorname{Spin}(n)$ on $\operatorname{Spin}(M) \times W_{j}$ by

$$
\operatorname{Spin}(n) \times\left(\operatorname{Spin}(M) \times W_{j}\right) \ni(g,(p, v)) \mapsto\left(p g^{-1}, \pi_{j}(g) v\right) \in \operatorname{Spin}(M) \times W_{j} .
$$

Then, we have a Hermitian vector bundle whose fiber is $W_{j}$,

$$
S_{j}:=\operatorname{Spin}(M) \times_{\operatorname{Spin}(n)} W_{j}=\left(\operatorname{Spin}(M) \times W_{j}\right) / \operatorname{Spin}(n) .
$$

For example, $S_{0}$ is the spinor bundle of $M$. We call a section of $S_{j}$ a spin $j+1 / 2$ field or $a$ spinor field with spin $j+1 / 2$.

From now on, we study some basic properties for the first-order differential operators naturally defined on the space of the spin $j+1 / 2$ fields $\Gamma\left(S_{j}\right)$. They are called generalized gradients or Stein-Weiss operators, which are defined by composing the orthogonal bundle projections and the covariant derivative $\nabla$ induced by the Levi-Civita connection, $[4,12]$. The covariant derivative on $\Gamma\left(S_{j}\right)$ is

$$
\nabla: \Gamma\left(S_{j}\right) \ni \phi \mapsto \nabla \phi=\sum \nabla_{e_{i}} \phi \otimes e_{i} \in \Gamma\left(S_{j} \otimes T M^{c}\right),
$$

where $\left\{e_{i}\right\}_{i}$ is a local orthonormal frame and $T M^{c}$ is $T M \otimes \mathbb{C} \cong T^{*} M \otimes \mathbb{C}$ by Riemannian metric $g$. We split the fiber $W_{j} \otimes \mathbb{C}^{n}$ into the sum of $\operatorname{Spin}(n)$-modules,

$$
W_{j} \otimes \mathbb{C}^{n}=W_{j+1} \oplus W_{j, 1} \oplus W_{j} \oplus W_{j-1},
$$

where $W_{j, 1}$ is a $\operatorname{Spin}(n)$-module with the highest weight

$$
\begin{cases}\left(j+1 / 2,3 / 2,(1 / 2)_{m-2}\right) \oplus\left(j+1 / 2,3 / 2,(1 / 2)_{m-3},-1 / 2\right) & \text { for } n=2 m, \\ \left(j+1 / 2,3 / 2,(1 / 2)_{m-3}\right) & \text { for } n=2 m-1 .\end{cases}
$$

We note that $W_{j, 1}$ does not appear for $n=3$ or $j=0$ and $W_{j-1}$ does not appear for $j=0$.

The space $W_{j}$ has a $\operatorname{Spin}(n)$-invariant Hermitian inner product (unique up to a constant factor), so that the above decomposition is orthogonal. Since the fiber metric on $S_{j}$ is induced from the inner product, we have the orthogonal bundle projection onto each irreducible summand. For example, composing $\nabla$ and the projection 


$$
\Pi_{j}: S_{j} \otimes T M^{c} \rightarrow S_{j}
$$

we have so-called the higher spin Dirac operator,

$$
\widetilde{D}_{j}:=\Pi_{j} \circ \nabla, \quad \Gamma\left(S_{j}\right) \stackrel{\nabla}{\longrightarrow} \Gamma\left(S_{j} \otimes T M^{c}\right) \stackrel{\Pi_{j}}{\longrightarrow} \Gamma\left(S_{j}\right) .
$$

In this manner, we construct four generalized gradients on $\Gamma\left(S_{j}\right)$ and name them as follows;

$$
\begin{array}{rr}
\widetilde{T}_{j}^{+}: \Gamma\left(S_{j}\right) \rightarrow \Gamma\left(S_{j+1}\right) & \text { the (first) twistor operator, } \\
U_{j}: \Gamma\left(S_{j}\right) \rightarrow \Gamma\left(S_{j, 1}\right) & \text { the (second) twistor operator, } \\
\widetilde{D}_{j}: \Gamma\left(S_{j}\right) \rightarrow \Gamma\left(S_{j}\right) & \text { the higher spin Dirac opeator, } \\
\widetilde{T}_{j}^{-}: \Gamma\left(S_{j}\right) \rightarrow \Gamma\left(S_{j-1}\right) & \text { the co-twistor opeator. }
\end{array}
$$

Here, we set $U_{j}=0$ for $n=3, U_{0}=0$ and $\widetilde{T}_{0}^{-}=0$. When $n=2 m$, each operator has the form of $2 \times 2$ matrix as

$$
\widetilde{D}_{j}=\left(\begin{array}{cc}
0 & * \\
* & 0
\end{array}\right), \quad \widetilde{T}_{j}^{+}, U_{j}, \widetilde{T}_{j}^{-}=\left(\begin{array}{cc}
* & 0 \\
0 & *
\end{array}\right)
$$

along the decomposition $\Gamma\left(S_{j}\right)=\Gamma\left(S_{j}^{+}\right) \oplus \Gamma\left(S_{j}^{-}\right)$. We introduce an $L^{2}$-inner product on $\Gamma\left(S_{j}\right)$ by

$$
\left(\phi_{1}, \phi_{2}\right):=\int_{M}\left\langle\phi_{1}, \phi_{2}\right\rangle \operatorname{vol}_{g}, \quad\left(\phi_{1}, \phi_{2} \in \Gamma\left(S_{j}\right)\right) .
$$

Then, the co-twistor operator $\widetilde{T}_{j}^{-}$is a nonzero constant multiple of the formal adjoint of the twistor operator $\widetilde{T}_{j-1}^{+}$with spin $j-1 / 2$,

$$
\left(\widetilde{T}_{j-1}^{+}\right)^{*}=c \widetilde{T}_{j}^{-}: \Gamma\left(S_{j}\right) \rightarrow \Gamma\left(S_{j-1}\right) .
$$

In the case of $j=0$, the operator $\widetilde{D}_{0}$ seems to coincide with the Dirac operator. However we know $\widetilde{D}_{0}=1 / \sqrt{n} D$. Therefore, we need a normalization of $\widetilde{D}_{j}$ to get the (usual) higher spin Dirac operator $D_{j}$ in other articles ([10] etc.). An advantage of our definition with projection is that we can apply Weitzenböck formulas in [16] and [17]. Furthermore such formulas allow us to give a normalization of $\widetilde{D}_{j}$ explicitly. Before normalizing the operators, we show some known results for analytic properties of the generalized gradients.

\section{Proposition 2.1}

1. $\widetilde{D}_{j}, \widetilde{T}_{j}^{ \pm}, U_{j}$ are conformally covariant.

2. $\widetilde{D}_{j}$ is a first-order, elliptic and (formally) self-adjoint differential operator.

3. $\widetilde{T}_{j}^{+}$is an overdetermined elliptic operator in the sense that the principal symbol $\sigma_{\xi, x}\left(\widetilde{T}_{j}^{+}\right)$ is injective for every $x$ in $M$ and nonzero covector $\xi$ in $T_{x}^{*} M$. Then, there is an orthogonal decomposition for the space of sections on a compact Riemannian spin manifold $M$,

$$
\Gamma\left(S_{j}\right)=\operatorname{ker}\left(\widetilde{T}_{j-1}^{+}\right)^{*} \oplus \widetilde{T}_{j-1}^{+}\left(\Gamma\left(S_{j-1}\right)\right)=\operatorname{ker} \widetilde{T}_{j}^{-} \oplus \widetilde{T}_{j-1}^{+}\left(\Gamma\left(S_{j-1}\right)\right) .
$$


Moreover, $\widetilde{T}_{j}^{+}$is a differential operator of finite type in the sense that the kernel of $\widetilde{T}_{j}^{+}$ is finite dimensional (even if $M$ is non-compact).

Proof The first claim follows from the fact that generalized gradient is conformally covariant $[12,16]$. As for the second claim, a general result for the ellipticity is known in $[4,22]$. We can also get the ellipticity for $D_{j}$ from Remark 2.13 . For the third one, we require a discussion. Let $U$ and $V$ be irreducible $\operatorname{Spin}(n)$-modules and $W$ be the Cartan summand of $U \otimes V$, the irreducible summand whose highest weight is the sum of those of $U$ and $V$. We consider the orthogonal projection $\Pi$ onto an irreducible summand of $U \otimes V$. Then, it is shown in [21] that the following two statements are equivalent:

- for $u \otimes v$ in $U \otimes V, u \otimes v \neq 0 \Rightarrow \Pi(u \otimes v) \neq 0$.

- $\Pi$ is the projection onto $W$.

Applying this to $U=W_{j}, V=\mathbb{C}^{n}=T_{x}^{*} M^{c}$ and $W=W_{j+1}$, we know $\Pi(u \otimes \xi) \neq 0$ for nonzero $u \otimes \xi$ in $W_{j} \otimes \mathbb{C}^{n}$. On the other hand, there is a result in [23] for finite type of differential equations such that this condition holds if and only if the generalized gradient is finite type. Thus, we have proved that $\widetilde{T}_{j}^{+}$is finite type and $\sigma_{\xi, x}\left(\widetilde{T}_{j}^{+}\right)$is injective. The decomposition of the space of sections with respect to an overdetermined elliptic operator is well known in [2]. Then, we get the decomposition of $\Gamma\left(S_{j}\right)$.

Remark 2.2 Let $S_{\rho}$ be an irreducible vector bundle with the highest weight $\rho=\left(\rho^{1}, \cdots, \rho^{m}\right)$. Then, we have the generalized gradient $D_{\rho+\mathbf{e}_{1}}^{\rho}$ from $S_{\rho}$ to the Cartan summand $S_{\rho+\mathbf{e}_{1}}$ in $S_{\rho} \otimes T M^{c}$ where $\rho+\mathbf{e}_{1}=\left(\rho^{1}+1, \rho^{2}, \ldots, \rho^{m}\right)$. In the same manner, the operator $D_{\rho+\mathbf{e}_{1}}^{\rho}$ is the only generalized gradient of finite type on $S_{\rho}$.

We shall give another definition of the higher spin Dirac operator by "twisting" the spinor bundle $S_{0}$ with the bundle of symmetric tensors in [10]. Let $\operatorname{Sym}^{j}=\operatorname{Sym}^{j}\left(T M^{c}\right)$ be the $j$-th symmetric tensor product bundle of $T M^{c}$ over $(M, g)$ and $\operatorname{Sym}_{0}^{j}$ be its primitive irreducible component whose fiber is an irreducible $\operatorname{Spin}(n)$-module with the highest weight $\left(j, 0_{m-1}\right)$. The bundle $\mathrm{Sym}^{j}$ is the direct sum of $\mathrm{Sym}_{0}^{j}$ and lower-order summands,

$$
\operatorname{Sym}^{j}=\operatorname{Sym}_{0}^{j} \oplus g \cdot \operatorname{Sym}_{0}^{j-2} \oplus g^{2} \cdot \operatorname{Sym}_{0}^{j-4} \oplus \cdots \oplus g^{[j / 2]} \cdot\left\{\begin{array}{l}
\operatorname{Sym}^{1} j \text { odd } \\
\operatorname{Sym}^{0} j \text { even, }
\end{array}\right.
$$

where $\operatorname{Sym}^{0}=\operatorname{Sym}_{0}^{0}$ is the trivial line bundle, $\operatorname{Sym}^{1}=\operatorname{Sym}_{0}^{1}$ is the tangent bundle $T M^{c}$, and $g^{k}$. is the symmetric tensor product of $g^{k}=g \cdots g$. We consider the tensor bundle $S_{0} \otimes \operatorname{Sym}_{0}^{j}$ and the twisted Dirac operator defined by

$$
D(j)=\sum_{k=1}^{n}\left(e_{k} \cdot \otimes \mathrm{id}_{\mathrm{Sym}_{0}^{j}}\right) \circ \nabla_{e_{k}} .
$$

Here, $\nabla$ is the covariant derivative on $S_{0} \otimes \operatorname{Sym}_{0}^{j}$, and $e_{k}$. is the Clifford multiplication by $e_{k}$. Along the decomposition of the bundle $S_{0} \otimes \operatorname{Sym}_{0}^{j}=S_{j} \oplus S_{j-1}$, we can realize $D(j)$ as a $2 \times 2$ matrix. As stated in [17], each component is a generalized gradient up to a nonzero multiplicative constant,

$$
D(j)=\left(\begin{array}{cc}
D_{j} & T_{j-1}^{+} \\
T_{j}^{-} & D_{j-1}^{\prime}
\end{array}\right), \quad \Gamma\left(S_{j} \oplus S_{j-1}\right) \rightarrow \Gamma\left(S_{j} \oplus S_{j-1}\right) .
$$


Since $D(j)$ is a formally self-adjoint operator, we get

$$
D_{j}^{*}=D_{j}, \quad\left(D_{j-1}^{\prime}\right)^{*}=D_{j-1}^{\prime}, \quad\left(T_{j-1}^{+}\right)^{*}=T_{j}^{-} .
$$

We will see that the square of $D(j)$ gives Weitzenböck formulas. From the twisted Lichnerowicz formula,

$$
D(j)^{2}=\Delta_{S_{0} \otimes \mathrm{Sym}_{0}^{j}}+\frac{\text { Scal }}{8}-\frac{1}{2} \mathrm{id}_{S_{0}} \otimes R_{\mathrm{Sym}_{0}^{j}} .
$$

We explain the first term $\Delta$ and the third term $R$ on the right side. First, we see the third term, the curvature action $R_{\operatorname{Sym}_{0}^{j}}$ on $\operatorname{Sym}_{0}^{j}\left(T M^{c}\right)$. Let $\left\{e_{i}=\left[p, \mathbf{e}_{i}\right]\right\}_{i=1}^{n}$ be a local orthonormal tangent frame of $T M=\mathrm{SO}(M) \times_{\mathrm{SO}(n)} \mathbb{R}^{n}$ and $\left\{e_{i j}=e_{i} \wedge e_{j}\right\}_{1 \leq i<j \leq n}$ be a local frame of

$$
\Lambda^{2}\left(T^{*} M\right) \simeq \mathfrak{o}(T M)=\mathfrak{g} \mathfrak{o}(T M)=\mathrm{SO}(M) \times_{\mathrm{SO}(n)} \mathfrak{g} \mathfrak{o}(n) .
$$

We have an action of the local section $e_{i j}$ of $\mathfrak{s} \mathfrak{p}(T M)$ on an associated vector bundle $S_{\rho}:=\operatorname{Spin}(M) \times_{\operatorname{Spin}(n)} W_{\rho}$ with respect to a (not necessarily irreducible) representation $\left(\pi_{\rho}, W_{\rho}\right)$ :

$$
\mathfrak{g o}(T M) \times S_{\rho} \ni\left(e_{i j}=\left[p, \mathbf{e}_{i} \wedge \mathbf{e}_{j}\right], \phi=[p, v]\right) \mapsto \pi_{\rho}\left(e_{i j}\right) \phi:=\left[p, \pi_{\rho}\left(\mathbf{e}_{i} \wedge \mathbf{e}_{j}\right) v\right] \in S_{\rho} .
$$

Then, we define the curvature action $R_{\rho}$ on $S_{\rho}$ by

$$
\begin{aligned}
R_{\rho} & =\frac{1}{2} \sum_{1 \leq i, j, k, l \leq n} R_{i j k l} \pi_{\rho}\left(e_{i j} e_{k l}\right) \\
& =\frac{1}{2} \sum_{1 \leq i, j, k, l \leq n} W_{i j k l} \pi_{\rho}\left(e_{i j} e_{k l}\right)+2 \sum_{1 \leq i, k, j \leq n} E_{i j} \pi_{\rho}\left(e_{i k} e_{j k}\right)+\frac{\pi_{\rho}\left(c_{2}\right)}{n(n-1)} \text { Scal } \\
& =: \text { Weyl }_{\rho}+\operatorname{Ein}_{\rho}+\text { Scal }_{\rho},
\end{aligned}
$$

where $R_{i j k l}=g\left(R\left(e_{i}, e_{j}\right) e_{k}, e_{l}\right)$ is the Riemannian curvature, $\left.\}\right\}$ Weyle the conformal Weyl tensor, \} \} Eine the traceless Ricci tensor and \}\}Scale the scalar curvature. The coefficient $\pi_{\rho}\left(c_{2}\right)$ of Scal is an action of the second Casimir element $c_{2}=\sum_{1 \leq i, j \leq n} e_{i j} e_{j i}$ on $W_{\rho}$, which acts on each irreducible bundle by a constant. Next we explain the first term of (4), the standard Laplacian or the Lichnerowicz Laplacian. On the bundle $S_{\rho}$, we define the second-order Laplace type operator by

$$
\Delta_{\rho}:=\nabla^{*} \nabla+\frac{1}{2} R_{\rho}
$$

This operatorcoincides with the Hodge Laplacian $\Delta=d d^{*}+d^{*} d$ on $S_{\rho}=\Lambda^{k}\left(T^{*} M\right)$ and $\Delta=D^{2}-\mathrm{Scal} / 8$ on the spinor bundle. On an irreducible compact symmetric space $G / K$, it corresponds to the second Casimir operator (nonnegative operator) for $G$. Remark that $\Delta_{\rho}$ is not necessarily nonnegative operator on a compact Riemannian manifold in general. One of important observation for the standard Laplacian is that, when we have a decomposition $S_{\rho}=S_{\lambda_{1}} \oplus \cdots \oplus S_{\lambda_{N}}$ as an associated vector bundle to $\operatorname{SO}(M)$ or $\operatorname{Spin}(M)$, the standard Laplacian $\Delta_{\rho}$ acts on each bundle diagonally (for more detail, [17, 23]).

We return to the case of $D(j)$. By the above observation, we have 


$$
D(j)^{2}=\left(\begin{array}{cc}
\Delta_{j}+\frac{\mathrm{Scal}}{8} & 0 \\
0 & \Delta_{j-1}+\frac{\mathrm{Scal}}{8}
\end{array}\right)-\frac{1}{2}\left(\begin{array}{cc}
\left(\mathrm{id} \otimes R_{\mathrm{Sym}_{0}^{j}}\right)_{j}^{j} & \left(\mathrm{id} \otimes R_{\mathrm{Sym}_{0}^{j}}\right)_{j}^{j-1} \\
\left(\mathrm{id} \otimes R_{\mathrm{Sym}_{0}^{j}}\right)_{j-1}^{j} & \left(\mathrm{id} \otimes R_{\mathrm{Sym}_{0}^{j}}\right)_{j-1}^{j-1}
\end{array}\right)
$$

along the decomposition $S_{j} \oplus S_{j-1}$. On the other hand, we take the square of the block matrix realization for $D(j)$,

$$
D(j)^{2}=\left(\begin{array}{cc}
D_{j}^{2}+T_{j-1}^{+} T_{j}^{-} & D_{j} T_{j-1}^{+}+T_{j-1}^{+} D_{j-1}^{\prime} \\
T_{j}^{-} D_{j}+D_{j-1}^{\prime} T_{j}^{-} & T_{j}^{-} T_{j-1}^{+}+\left(D_{j-1}^{\prime}\right)^{2}
\end{array}\right) .
$$

Comparing the above two equations, we show

$$
\begin{gathered}
D_{j}^{2}+T_{j-1}^{+}\left(T_{j-1}^{+}\right)^{*}=\Delta_{j}+\frac{\mathrm{Scal}}{8}-\frac{1}{2}\left(\mathrm{id} \otimes R_{\mathrm{Sym}_{0}^{j}}\right)_{j}^{j}, \\
\left(T_{j-1}^{+}\right)^{*} T_{j-1}^{+}+\left(D_{j-1}^{\prime}\right)^{2}=\Delta_{j-1}+\frac{\mathrm{Scal}}{8}-\frac{1}{2}\left(\mathrm{id} \otimes R_{\mathrm{Sym}_{0}^{j}}\right)_{j-1}^{j-1}, \\
\left(T_{j-1}^{+}\right)^{*} D_{j}+D_{j-1}^{\prime}\left(T_{j-1}^{+}\right)^{*}=-\frac{1}{2}\left(\mathrm{id} \otimes R_{\mathrm{Sym}_{0}^{j}}\right)_{j-1}^{j}, \\
D_{j} T_{j-1}^{+}+T_{j-1}^{+} D_{j-1}^{\prime}=-\frac{1}{2}\left(\mathrm{id} \otimes R_{\mathrm{Sym}_{0}^{j}}\right)_{j}^{j-1} .
\end{gathered}
$$

The curvature term (id $\left.\otimes R_{\mathrm{Sym}_{0}^{j}}\right)_{j-1}^{j}$ is a bundle homomorphism from $S_{j}$ to $S_{j-1}$ depending only on the conformal Weyl tensor part $\mathrm{Weyl}_{\mathrm{Sym}_{0}^{j}}$ and the traceless Ricci tensor part $\operatorname{Ein}_{\mathrm{Sym}_{0}^{j}}$ ([17]). Remark that, in the case of $j=1$, it depends only on the traceless Ricci tensor, so that there are fruitful result of the Rarita-Schwinger operator on an Einstein manifold in [18]. On the other hand, the curvature term (id $\left.\otimes R_{\mathrm{Sym}_{0}^{j}}\right)_{j}^{j}$ on $S_{j}$ depends not only on Weyl $_{\mathrm{Sym}_{0}^{j}}$ and $\operatorname{Ein}_{\mathrm{Sym}_{0}^{j}}$ but also on the scalar curvature part. Therefore, we can calculate the curvature terms explicitly on a Riemannian manifold of constant sectional curvature $K=c$ and obtain the following proposition.

Proposition 2.3 If $(M, g)$ is a Riemannian manifold of constant sectional curvature $K=c$ with a spin structure, then

$$
\begin{gathered}
D_{j}^{2}+T_{j-1}^{+}\left(T_{j-1}^{+}\right)^{*}=\Delta_{j}-\left(j(n+j-2)-\frac{n(n-1)}{8}\right) c, \\
\left(T_{j-1}^{+}\right)^{*} T_{j-1}^{+}+\left(D_{j-1}^{\prime}\right)^{2}=\Delta_{j-1}-\left(j(n+j-2)-\frac{n(n-1)}{8}\right) c, \\
\left(T_{j-1}^{+}\right)^{*} D_{j}+D_{j-1}^{\prime}\left(T_{j-1}^{+}\right)^{*}=0, \quad D_{j} T_{j-1}^{+}+T_{j-1}^{+} D_{j-1}^{\prime}=0 .
\end{gathered}
$$

Proof The conformal Weyl tensor and the traceless Ricci tensor are zero, and the scalar curvature is $\mathrm{Scal}=n(n-1) c$ on $(M, g)$. The action of $-\frac{1}{2} \mathrm{id} \otimes R_{\mathrm{Sym}_{0}^{j}}$ is constant by

$$
-\frac{\pi_{\mathrm{Sym}_{0}^{j}}\left(c_{2}\right)}{2 n(n-1)} \mathrm{Scal}=-j(n+j-2) c .
$$

Then, we have 


$$
D_{j}^{2}+T_{j-1}^{+} T_{j}^{-}=\Delta_{j}-\left(j(n+j-2)-\frac{n(n-1)}{8}\right) c .
$$

Similarly we prove (7) and (8).

Furthermore, because of $D(j)^{2} D(j)=D(j) D(j)^{2}$, we have

$$
\left(\begin{array}{cc}
\Delta_{j}+c^{\prime} & 0 \\
0 & \Delta_{j-1}+c^{\prime}
\end{array}\right)\left(\begin{array}{cc}
D_{j} & T_{j-1}^{+} \\
T_{j}^{-} & D_{j-1}^{\prime}
\end{array}\right)=\left(\begin{array}{cc}
D_{j} & T_{j-1}^{+} \\
T_{j}^{-} & D_{j-1}^{\prime}
\end{array}\right)\left(\begin{array}{cc}
\Delta_{j}+c^{\prime} & 0 \\
0 & \Delta_{j-1}+c^{\prime}
\end{array}\right),
$$

where $c^{\prime}=-\left(j(n+j-2)-\frac{n(n-1)}{8}\right) c$. Then, we conclude that

$$
\Delta_{j} D_{j}=D_{j} \Delta_{j}, \quad T_{j-1}^{+} \Delta_{j-1}=\Delta_{j} T_{j-1}^{+}, \quad T_{j}^{-} \Delta_{j}=\Delta_{j-1} T_{j}^{-}
$$

on a space of constant curvature. Note that these commutation relations hold on a locally symmetric space $(M, g)$ because its curvature $R$ satisfies $\nabla R=0$ (see Proposition 8 in [17]).

Now we calculate a normalizing constant $c_{j}$ in the equation $D_{j}=c_{j} \widetilde{D}_{j}$. Thanks to Weitzenböck formulas in [16] (see Appendix A for more detail), the generalized gradients $\left\{\widetilde{D}_{j}, \widetilde{T}_{j}^{ \pm}\right\}_{j}$ satisfy

$$
\begin{gathered}
\frac{(n+2 j)(n-2)}{n+2 j-2} \widetilde{D}_{j}^{2}+\frac{4(n+j-2)}{n+2 j-2}\left(\widetilde{T}_{j}^{-}\right)^{*} \widetilde{T}_{j}^{-}=\Delta_{j}+\text { curv, } \\
\frac{4 j}{n+2 j-2}\left(\widetilde{T}_{j-1}^{+}\right)^{*} \widetilde{T}_{j-1}^{+}+\frac{(n+2 j-4)(n-2)}{n+2 j-2}\left(\widetilde{D}_{j-1}\right)^{2}=\Delta_{j-1}+\text { curv, }
\end{gathered}
$$

where "curv" means a bundle endomorphism depending on the Riemannian curvature as before. We also know that $D_{j}^{2}$ and $T_{j-1}^{+} T_{j}^{-}$are independent in the sense that there is no relation of the form

$$
a D_{j}^{2}+b T_{j-1}^{+} T_{j}^{-}=\mathrm{curv}, \quad(a, b) \neq(0,0)
$$

and so are $D_{j}^{2}$ and $T_{j+1}^{-} T_{j}^{+}$. Then, comparing the equations (6), (7), (9) and (10), we obtain the normalizing constant for $D_{j}$ with respect to $\widetilde{D}_{j}=\Pi_{j} \circ \nabla$ in the next proposition.

Proposition 2.4 We consider a Riemannian spin manifold $(M, g)$ and the vector bundle $S_{j}=\operatorname{Spin}(M) \times_{\operatorname{Spin}(n)} W_{j}$ with spin $j+1 / 2$. Let $\widetilde{D}_{j}=\Pi_{j} \circ \nabla$ be the generalized gradient on $S_{j}$ defined by (1), and $D_{j}$ (resp. $\left.D_{j-1}^{\prime}\right)$ be the operator defined by restricting the twisted Dirac operator $D(j)$ on $S_{j}$ (resp. on $S_{j-1}$ ) in (3). Then,

$$
D_{j}=\sqrt{\frac{(n+2 j)(n-2)}{n+2 j-2}} \widetilde{D}_{j}, \quad D_{j-1}^{\prime}=-\sqrt{\frac{(n+2(j-1)-2)(n-2)}{n+2(j-1)}} \widetilde{D}_{j-1},
$$

and hence,

$$
D_{j}^{2}=\frac{(n+2 j)(n-2)}{n+2 j-2} \tilde{D}_{j}^{2}, \quad\left(D_{j-1}^{\prime}\right)^{2}=\frac{(n+2(j-1)-2)(n-2)}{n+2(j-1)} \tilde{D}_{j-1}^{2} .
$$

As a result, we have 


$$
D_{j}^{\prime}=-\frac{n+2 j-2}{n+2 j} D_{j}
$$

We also have normalizing constants for the twistor operators $T_{j}^{ \pm}$on $S_{j}$,

$$
T_{j}^{-}=2 \sqrt{\frac{n+j-2}{n+2 j-2}} \widetilde{T}_{j}^{-}, \quad T_{j}^{+}=2 \sqrt{\frac{j+1}{n+2 j}} \widetilde{T}_{j}^{+} .
$$

Hence, we have

$$
\begin{aligned}
\nabla^{*} \nabla & =\left(\widetilde{T}_{j}^{+}\right)^{*} \widetilde{T}_{j}^{+}+U_{j}^{*} U_{j}+\widetilde{D}_{j}^{2}+\left(\widetilde{T}_{j}^{-}\right)^{*} \widetilde{T}_{j}^{-}, \\
& =\frac{n+2 j}{4(j+1)}\left(T_{j}^{+}\right)^{*} T_{j}^{+}+U_{j}^{*} U_{j}+\frac{n+2 j-2}{(n+2 j)(n-2)} D_{j}^{2}+\frac{n+2 j-2}{4(n+j-2)}\left(T_{j}^{-}\right)^{*} T_{j}^{-} .
\end{aligned}
$$

Remark 2.5 The generalized gradient is defined up to constant multiple of a complex number $u$ with norm $|u|=1$, so that the above normalizing constant is unique up to such $u$. In the case of $D_{j}$ (resp. $D_{j}^{\prime}$ ), taking into account the self-adjointness, we put $u=1$ (resp. $u=-1)$.

Remark 2.6 By the homomorphism type Weitzenböck formula given in [17], we can give an alternative proof of the calculation of the normalizing constant for $D_{j}^{\prime}=c D_{j}$. In fact, we have

$$
\frac{1}{\sqrt{n / 2+j-2}} \widetilde{D}_{j} \widetilde{T}_{j-1}^{+}=\frac{1}{\sqrt{n / 2+j}} \widetilde{T}_{j-1}^{+} \widetilde{D}_{j-1} .
$$

Therefore,

$$
D_{j} T_{j-1}^{+}=\frac{n+2 j-4}{n+2 j-2} T_{j-1}^{+} D_{j-1} .
$$

Then, we have concluded $D_{j}^{\prime}=-\frac{n+2 j-2}{n+2 j} D_{j}$.

Example 2.7 The operator $D_{0}$ is just the Dirac operator $D$. The operator $D_{1}$ is the RaritaSchwinger operator $Q$ in [18].

As a corollary of Proposition 2.3 and 2.4, we rewrite the Weitzenböck formula in terms of $D_{j}$ and $T_{j}^{ \pm}$, which will be useful in later calculations.

Corollary 2.8 Let $(M, g)$ be a space of constant curvature $K=c$ with a spin structure. Then, the following identities for operators from $\Gamma\left(S_{j}\right)$ to $\Gamma\left(S_{j}\right)$ hold:

$$
\begin{aligned}
\Delta_{j} & =\nabla^{*} \nabla+\frac{1}{2} R_{j}=\nabla^{*} \nabla+\left(j(n+j-1)+\frac{n(n-1)}{8}\right) c \\
& =D_{j}^{2}+\left(T_{j}^{-}\right)^{*} T_{j}^{-}+\left(j(n+j-2)-\frac{n(n-1)}{8}\right) c \\
& =\left(T_{j}^{+}\right)^{*} T_{j}^{+}+\frac{(n+2 j-2)^{2}}{(n+2 j)^{2}} D_{j}^{2}+\left((j+1)(n+j-1)-\frac{n(n-1)}{8}\right) c .
\end{aligned}
$$

The generalized gradients among $\Gamma\left(S_{j-1}\right)$ and $\Gamma\left(S_{j}\right)$ are related as follows: 


$$
T_{j}^{-} D_{j}=\frac{n+2 j-4}{n+2 j-2} D_{j-1} T_{j}^{-}, \quad D_{j} T_{j-1}^{+}=\frac{n+2 j-4}{n+2 j-2} T_{j-1}^{+} D_{j-1}, \quad T_{j-1}^{+}=\left(T_{j}^{-}\right)^{*} .
$$

The main theorem in this section is a factorization formula for the power of the Laplacian on a space of constant curvature. On the flat space $\mathbb{R}^{n}$, it is known in $[9,10]$ and $[11]$ that $D_{j}$ factors through $\left(\Delta_{j}\right)^{j+1}$. In other words, there is a differential operator $A_{2 j+1}$ with order $2 j+1$ such that $\left(\Delta_{j}\right)^{j+1}=D_{j} \circ A_{2 j+1}$. It follows that the known fundamental solution $G$ for $\left(\Delta_{j}\right)^{j+1}$ gives a fundamental solution $A_{2 j+1}(G)$ for $D_{j}$. We can generalize this factorization on $\mathbb{R}^{n}$ to a space of constant curvature explicitly.

Theorem 2.9 (Factorization formula) Let $(M, g)$ be a space of constant curvature $K=c$ with a spin structure. For the higher spin Dirac operator $D_{j}$ and the standard Laplacian $\Delta_{j}$ on $S_{j}$, we define a second-order operator on $\Gamma\left(S_{j}\right)$,

$$
B(s ; j):=D_{j}^{2}-\frac{(n+2 s-2)^{2}}{(n+2 j-2)^{2}}\left(\Delta_{j}-\left(s(n+s-2)-\frac{n(n-1)}{8}\right) c\right)
$$

for $s=0,1, \cdots, j+1$. Then, we have

$$
\prod_{s=0}^{j} B(s ; j)=0 .
$$

Proof We prove the theorem by induction for $j$. We start from the equation for $j=0$,

$$
\prod_{s=0}^{0} B(s ; 0)=B(0 ; 0)=D_{0}^{2}-\left(\Delta_{0}+\frac{n(n-1)}{8} c\right)=0 .
$$

This is just the Lichnerowicz formula for the Dirac operator. We assume the equation (13) holds for $j$. From (12), we easily show the key relations

$$
\begin{gathered}
T_{j}^{+} B(s ; j)=\frac{(n+2 j)^{2}}{(n+2 j-2)^{2}} B(s ; j+1) T_{j}^{+}, \\
T_{j+1}^{-} B(s ; j+1)=\frac{(n+2 j-2)^{2}}{(n+2 j)^{2}} B(s ; j) T_{j+1}^{-}, \\
\left(T_{j}^{-}\right)^{*} T_{j}^{-}=-B(j ; j), \quad\left(T_{j}^{+}\right) T_{j}^{+}=-\frac{(n+2 j-2)^{2}}{(n+2 j)^{2}} B(j+1 ; j) .
\end{gathered}
$$

Sandwich (13) by $T_{j}^{+}$and $\left(T_{j}^{+}\right)^{*}$, and we obtain

$$
\begin{aligned}
0 & =T_{j}^{+}\left(\prod_{s=0}^{j} B(s ; j)\right)\left(T_{j}^{+}\right)^{*}=\left(\prod_{s=0}^{j} \frac{(n+2 j)^{2}}{(n+2 j-2)^{2}} B(s ; j+1)\right) T_{j}^{+}\left(T_{j}^{+}\right)^{*}, \\
& =-\frac{(n+2 j)^{2(j+1)}}{(n+2 j-2)^{2(j+1)}}\left(\prod_{s=0}^{j} B(s ; j+1)\right) B(j+1 ; j+1) .
\end{aligned}
$$

Thus, we have proved (13) holds for $j+1$.

To clarify the meaning of the above factorization formula, we introduce a filtration on $\Gamma\left(S_{j}\right)$. Put 


$$
F_{j}:=\operatorname{ker} T_{j}^{-}, \quad F_{j-1}:=\operatorname{ker} T_{j-1}^{-} T_{j}^{-}, \quad \cdots \quad F_{1}:=\operatorname{ker} T_{1}^{-} \cdots T_{j}^{-}, \quad F_{0}:=\Gamma\left(S_{j}\right),
$$

and we have a filtration on $\Gamma\left(S_{j}\right)$,

$$
F_{j} \subset F_{j-1} \subset \cdots \subset F_{1} \subset F_{0}=\Gamma\left(S_{j}\right) .
$$

Proposition 2.10 On a compact space $(M, g)$ of constant curvature $K=c$ with a spin structure,

$$
F_{j}=\operatorname{ker} B(j ; j), \quad \cdots, \quad F_{k}=\operatorname{ker} \prod_{s=k}^{j} B(s ; j), \quad \cdots, \quad F_{0}=\Gamma\left(S_{j}\right),
$$

for $0 \leq k \leq j$.

Proof If $\phi$ is in $F_{k}$, then $\left(T_{j}^{-}\right)^{*} \cdots\left(T_{k}^{-}\right)^{*} T_{k}^{-} \cdots T_{j}^{-} \phi=0$. Conversely, if

$$
\left(T_{j}^{-}\right)^{*} \cdots\left(T_{k}^{-}\right)^{*} T_{k}^{-} \cdots T_{j}^{-} \phi=0,
$$

then

$$
\begin{aligned}
0 & =\int_{M}\left\langle\left(T_{j}^{-}\right)^{*} \cdots\left(T_{k}^{-}\right)^{*} T_{k}^{-} \cdots T_{j}^{-} \phi, \phi\right\rangle \operatorname{vol}_{g} \\
& =\int_{M}\left|T_{k}^{-} \cdots T_{j}^{-} \phi\right|^{2} \operatorname{vol}_{g}=\left\|T_{k}^{-} \cdots T_{j}^{-} \phi\right\|^{2} .
\end{aligned}
$$

Thus, $\phi$ is in $F_{k}$ if and only if $\left(T_{j}^{-}\right)^{*} \cdots\left(T_{k}^{-}\right)^{*} T_{k}^{-} \cdots T_{j}^{-} \phi=0$. From (14),

$$
\begin{aligned}
\left(T_{j}^{-}\right)^{*} \cdots\left(T_{k}^{-}\right)^{*} T_{k}^{-} \cdots T_{j}^{-}= & -\left(T_{j}^{-}\right)^{*} \cdots\left(T_{k+1}^{-}\right)^{*} B(k ; k) T_{k+1}^{-} \cdots T_{j}^{-} \\
= & c B(k ; j)\left(T_{j}^{-}\right)^{*} \cdots\left(T_{k+1}^{-}\right)^{*} T_{k+1}^{-} \cdots T_{j}^{-} \quad(\exists c \neq 0) \\
= & -c B(k ; j)\left(T_{j}^{-}\right)^{*} \cdots\left(T_{k+2}^{-}\right)^{*} B(k+1 ; k+1) T_{k+2}^{-} \cdots T_{j}^{-} \\
& \cdots \\
= & c^{\prime} B(k ; j) \cdots B(j ; j), \quad\left(\exists c^{\prime} \neq 0\right) .
\end{aligned}
$$

Then, we can prove the proposition.

The above natural filtration allows us to get an associated grading on $\Gamma\left(S_{j}\right)$. In other words, there are subspaces $W_{s}$ for $s=0, \cdots, j$ such that $F_{s}=W_{s} \oplus F_{s+1}$ and $\Gamma\left(S_{j}\right)=\bigoplus_{0 \leq s \leq j} W_{s}$.

Theorem 2.11 Let $S_{j}$ be the bundle of spinor fields with spin $j+1 / 2$ on a compact space of constant curvature $K=c$ with a spin structure. We put

$$
W_{s}:= \begin{cases}T_{j-1}^{+} \cdots T_{0}^{+}\left(\Gamma\left(S_{0}\right)\right), & s=0, \\ T_{j-1}^{+} \cdots T_{s}^{+}\left(\operatorname{ker} T_{s}^{-}\right), & 1 \leq s \leq j-1, \\ \operatorname{ker} T_{j}^{-}, & s=j .\end{cases}
$$

Then, the space $\Gamma\left(S_{j}\right)$ has an orthogonal decomposition associated with the filtration $\left\{F_{j}\right\}_{j}$, 


$$
\Gamma\left(S_{j}\right)=\bigoplus_{0 \leq s \leq j} W_{s}, \quad F_{s}=W_{s} \oplus F_{s+1} \quad(0 \leq s \leq j) .
$$

Furthermore, $W_{s} \subset \operatorname{ker} B(s ; j)$ for $s=0, \cdots, j$.

Proof From Proposition 2.1, there are the orthogonal decompositions

$$
\Gamma\left(S_{s}\right)=\operatorname{ker} T_{s}^{-} \oplus T_{s-1}^{+}\left(\Gamma\left(S_{s-1}\right)\right)
$$

for $s=0,1, \cdots$. When we let $T_{s}^{+}$act on $\Gamma\left(S_{s}\right)$, we can prove that the decomposition is preserved,

$$
T_{s}^{+}\left(\Gamma\left(S_{s}\right)\right)=T_{s}^{+}\left(\operatorname{ker} T_{s}^{-}\right) \oplus T_{s}^{+} T_{s-1}^{+}\left(\Gamma\left(S_{s-1}\right)\right) .
$$

Since $T_{s}^{+}\left(\Gamma\left(S_{s}\right)\right)=T_{s}^{+}\left(\operatorname{ker} T_{s}^{-}\right)+T_{s}^{+} T_{s-1}^{+}\left(\Gamma\left(S_{s-1}\right)\right)$, all we have to do is to prove that the components intersect orthogonally. Take $\phi_{s}$ in $\operatorname{ker} T_{s}^{-}$, and we show

$$
\begin{aligned}
T_{s}^{-} T_{s+1}^{-} T_{s}^{+}\left(\phi_{s}\right) & =T_{s}^{-}\left(T_{s}^{+}\right)^{*} T_{s}^{+} \phi_{s}=-\frac{(n+2 s-2)^{2}}{(n+2 s)^{2}} T_{s}^{-} B(s+1 ; s) \phi_{s} \\
& =-\frac{(n+2 s-4)^{2}}{(n+2 s)^{2}} B(s+1 ; s-1) T_{s}^{-} \phi_{s}=0 .
\end{aligned}
$$

Then,

$$
\left(T_{s}^{+} \phi_{s}, T_{s}^{+} T_{s-1}^{+} \psi_{s-1}\right)=\left(T_{s}^{-} T_{s+1}^{-} T_{s}^{+} \phi_{s}, \psi_{s-1}\right)=0 \quad \text { for } \psi_{s-1} \text { in } \Gamma\left(S_{s-1}\right) \text {. }
$$

Thus, we have obtained an orthogonal decomposition $T_{s}^{+}\left(\Gamma\left(S_{s}\right)\right)=T_{s}^{+}\left(\operatorname{ker} T_{s}^{-}\right) \oplus T_{s}^{+} T_{s-1}^{+}\left(\Gamma\left(S_{s-1}\right)\right)$. As a consequence, we have

$$
\begin{array}{r}
\Gamma\left(S_{s+1}\right)=\operatorname{ker} T_{s+1}^{-} \oplus T_{s}^{+}\left(\operatorname{ker} T_{s}^{-}\right) \oplus T_{s}^{+} T_{s-1}^{+}\left(\Gamma\left(S_{s-1}\right)\right), \\
F_{s+1}=\operatorname{ker} T_{s+1}^{-}, \quad F_{s}=\operatorname{ker} T_{s+1}^{-} \oplus T_{s}^{+}\left(\operatorname{ker} T_{s}^{-}\right) .
\end{array}
$$

Repeating this, we have concluded $\Gamma\left(S_{j}\right)=\oplus_{s} W_{s}$.

Next we prove $W_{s} \subset \operatorname{ker} B(s ; j)$. For $\phi_{s}$ in $\operatorname{ker} T_{s}^{-}$,

$$
\begin{aligned}
B(s ; j) T_{j-1}^{+} \cdots T_{s}^{+} \phi_{s} & =\frac{(n+2 j-4)^{2}}{(n+2 j-2)^{2}} T_{j-1}^{+} B(s ; j-1) T_{j-2}^{+} \cdots T_{s}^{+} \phi_{s} \\
& =\frac{(n+2 s-2)^{2}}{(n+2 j-2)^{2}} T_{j-1}^{+} \cdots T_{s+1}^{+} T_{s}^{+} B(s ; s) \phi_{s} \\
& =-\frac{(n+2 s-2)^{2}}{(n+2 j-2)^{2}} T_{j-1}^{+} \cdots T_{s+1}^{+} T_{s}^{+}\left(T_{s}^{-}\right)^{*} T_{s}^{-} \phi_{s}=0 .
\end{aligned}
$$

Thus, we have concluded that $W_{s} \subset \operatorname{ker} B(s ; j)$. Remark that the reverse inclusion does not hold in general (for example, the case of $K=0$ ).

Since $D_{j} \Delta_{j}=\Delta_{j} D_{j}$, the operator $D_{j}$ and $\Delta_{j}$ have simultaneous eigenstates on a compact space $(M, g)$ of constant curvature and their eigenvalues relate to each other. Let $\phi$ is a simultaneous eigenspinor in $W_{k}$ with eigenvalue $\mu$ for $\Delta_{j}$ and $\lambda^{2}$ for $D_{j}^{2}$. Then, 


$$
\lambda^{2}=\frac{(n+2 k-2)^{2}}{(n+2 j-2)^{2}}\left(\mu-\left(k(n+k-2)-\frac{n(n-1)}{8}\right) c\right)
$$

For the non-compact case, the situation is more difficult. In fact, it has already been known that $\Delta_{j} \phi=0$ does not follow $D_{j} \phi=0$ on $\mathbb{R}^{n}$, and vice versa ([10]).

At the end of this section, we state some remarks.

Remark 2.12 It was shown in [18] that the Rarita-Schwinger operator $D_{1}$ satisfies

$$
\left(D_{1}^{2}-\left(\Delta_{1}+\frac{n-8}{8 n} \text { Scal }\right)\right)\left(D_{1}^{2}-\frac{(n-2)^{2}}{n^{2}}\left(\Delta_{1}+\frac{\text { Scal }}{8}\right)\right)=0
$$

on an Einstein manifold $(M, g)$. To get a factorization formula for higher spin cases of $j>1$, we have to assume the sectional curvature is constant because the curvature term and their derivatives remain in Weitzenböck formulas and the formula is too complicated to get a factorization. To calculate such error terms is a future problem.

Remark 2.13 The above theorem shows that $\sigma_{\xi}\left(D_{j}\right)$ is a factor of $\sigma_{\xi}\left(\Delta_{j}^{j+1}\right)=\left(-\|\xi\|^{2}\right)^{j+1}$ on the principal symbol level, and so that $D_{j}$ is elliptic.

Remark 2.14 We can rewrite the equation (13) for $T_{j}^{+}$as

$$
\prod_{s=0}^{j}\left(\left(T_{j}^{+}\right)^{*} T_{j}^{+}-a^{\prime}(s ; j)\left(\Delta_{j}-b^{\prime}(s ; j) c\right)\right)=0,
$$

where

$$
a^{\prime}(s ; j)=\frac{4(j-s+1)(n+j+s-1)}{(n+2 j)^{2}}, \quad b^{\prime}(s ; j)=j(n+j)+s(n+s-2)+\frac{n(n+1)}{8} .
$$

Then, the eigenvalue of the standard Laplacian $\Delta_{j}$ restricted to $\operatorname{ker} T_{j}^{+} \cap W_{k}$ on a compact space of constant curvature is just

$$
\left(j(n+j)+k(n+k-2)+\frac{n(n+1)}{8}\right) c .
$$

\section{Harmonic analysis for spinor fields with higher spin on $S^{n}$}

In this section, we study harmonic analysis for spin $j+1 / 2$ fields on the sphere. We calculate the eigenvalues of $D_{j}^{2}$ and other related second-order operators and show how the space of spinor fields are related to each other as $\operatorname{Spin}(n+1)$-modules.

Let $S^{n}$ be the $n$-dimensional standard sphere. We realize it as a $\operatorname{Spin}(n+1)$-orbit of $e_{n+1}=(0, \cdots, 0,1)$ in $\mathbb{R}^{n+1}$. The isotropy group is

$$
\operatorname{Spin}(n)=\left\{g \in \operatorname{Spin}(n+1) \mid g \cdot e_{n+1}=e_{n+1}\right\},
$$

and the sphere is a symmetric $\operatorname{space} \operatorname{Spin}(n+1) / \operatorname{Spin}(n)$. The (unique) spin structure of $S^{n}$ is the principal $\operatorname{Spin}(n)$-bundle $\operatorname{Spin}(n+1)$ where $\operatorname{Spin}(n)$ acts naturally from the right side. The bundle of higher spinor fields is given by 


$$
S_{j}=\operatorname{Spin}(n+1) \times_{\operatorname{Spin}(n)} W_{j}
$$

with a natural fiber metric induced from a $\operatorname{Spin}(n)$-invariant inner product on $W_{j}$. The space $L^{2}\left(S^{n}, S_{j}\right)$ of $L^{2}$-integrable sections of $S_{j}$ is a unitary representation space of $\operatorname{Spin}(n+1)$ with respect to $L^{2}$-inner product $(\phi, \psi)=\int_{S^{n}}\langle\phi, \psi\rangle \operatorname{vol}_{g}$. Frobenius reciprocity provides its irreducible decomposition,

$$
L^{2}\left(S^{n}, S_{j}\right) \cong \bigoplus_{\rho \in \operatorname{Spin}(n+1)} V_{\rho} \otimes \operatorname{Hom}_{\operatorname{Spin}(n)}\left(V_{\rho}, W_{j}\right),
$$

where $\widehat{\operatorname{Spin}(n+1)}$ means the equivalent classes of irreducible unitary finite dimensional representations of $\operatorname{Spin}(n+1)$. Such a class is parametrized by its highest weight $\rho=\left(\rho^{1}, \ldots, \rho^{m}\right)$ for $m=\left[\frac{n+1}{2}\right]$ satisfying $\rho$ in $\mathbb{Z}^{m} \cup(\mathbb{Z}+1 / 2)^{m}$ and the dominant condition

$$
\begin{gathered}
\rho^{1} \geq \rho^{2} \geq \cdots \geq \rho^{m} \geq 0, \quad(\text { for } n+1=2 m+1), \\
\rho^{1} \geq \rho^{2} \geq \cdots \geq \rho^{m-1} \geq\left|\rho^{m}\right|, \quad(\text { for } n+1=2 m) .
\end{gathered}
$$

We state the branching rule to describe the restriction of an irreducible representation of $\operatorname{Spin}(n+1)$ to $\operatorname{Spin}(n)$ (cf. [27]). Fix an irreducible $\operatorname{Spin}(n+1)$-module $V_{\rho}$ with the highest weight $\rho$. When $V_{\rho}$ is considered as a $\operatorname{Spin}(n)$-module, the following $\operatorname{Spin}(n)$-modules appear each with multiplicity 1 as irreducible summands:

- In the case of $n+1=2 m+1, \lambda=\left(\lambda^{1}, \cdots, \lambda^{m}\right)$ in $\left.\widehat{\operatorname{Spin}(2 m}\right)$ satisfying

$$
\rho^{1} \geq \lambda^{1} \geq \rho^{2} \geq \lambda^{2} \geq \cdots \geq \rho^{m} \geq \lambda^{m} \geq-\rho^{m}, \quad \rho^{1}-\lambda^{1} \in \mathbb{Z} .
$$

- In the case of $n+1=2 m, \lambda=\left(\lambda^{1}, \cdots, \lambda^{m-1}\right)$ in Spin( $\left.\widehat{(2 m}-1\right)$ satisfying

$$
\rho^{1} \geq \lambda^{1} \geq \rho^{2} \geq \lambda^{2} \geq \cdots \geq \rho^{m-1} \geq \lambda^{m-1} \geq\left|\rho^{m}\right|, \quad \rho^{1}-\lambda^{1} \in \mathbb{Z} .
$$

We denote by $V_{j}(k, s)$ an irreducible $\mathrm{SO}(n+1)$-module with the highest weight

$$
\left(k+j, s, 0_{m-2}\right)
$$

for $k$ in $\mathbb{Z}_{\geq 0}$ and $s=0, \ldots, j$. We also denote by $V_{j}(k, s)^{\prime}$ an irreducible $\operatorname{Spin}(2 m+1)$-module with the highest weight

$$
\left(k+j+1 / 2, s+1 / 2,(1 / 2)_{m-2}\right),
$$

and by $V_{j}^{ \pm}(k, s)^{\prime}$ an irreducible $\operatorname{Spin}(2 m)$-module with the highest weight

$$
\left(k+j+1 / 2, s+1 / 2,(1 / 2)_{m-3}, \pm 1 / 2\right)
$$

for $k$ in $\mathbb{Z}_{\geq 0}$ and $s=0, \ldots, j$.

Remark 3.1 In the case of $n+1=4$, the space $V_{j}(k, s)$ splits into the sum of $\mathrm{SO}(4)$-modules with the highest weight $(k+j, s)$ and $(k+j,-s)$. The space $V_{j}^{ \pm}(k, s)^{\prime}$ means an irreducible Spin(4)-module with the highest weight $(k+j+1 / 2, \pm(s+1 / 2))$.

Applying Frobenius reciprocity and the branching rule to our case $\lambda=(j+1 / 2,1 / 2, \cdots, 1 / 2)$, we have the proposition. 


\section{Proposition 3.2}

1. For $n=2 m$,

$$
L^{2}\left(S^{2 m}, S_{j}^{ \pm}\right) \cong \bigoplus_{0 \leq s \leq j} \bigoplus_{k \geq 0} V_{j}(k, s)^{\prime}, \quad L^{2}\left(S^{2 m}, S_{j}\right) \cong \bigoplus_{0 \leq s \leq j} \bigoplus_{k \geq 0} 2 V_{j}(k, s)^{\prime} .
$$

2. For $n=2 m-1$,

$$
L^{2}\left(S^{2 m-1}, S_{j}\right) \cong \bigoplus_{0 \leq s \leq j} \bigoplus_{k \geq 0} V_{j}^{+}(k, s)^{\prime} \oplus V_{j}^{-}(k, s)^{\prime} .
$$

From now on, we put $V_{j}(k, s)^{\prime}=V_{j}^{+}(k, s)^{\prime} \oplus V_{j}^{-}(k, s)^{\prime}$ for $n=2 m-1$. By Weyl's dimension formula, we have

$$
\operatorname{dim} V_{j}(k, s)^{\prime}=2^{\left[\frac{n+1}{2}\right]} \frac{(l+n-1+s)(l+1-s)}{(n-1)(n-2)}\left(\begin{array}{c}
l+n-2 \\
l+1
\end{array}\right)\left(\begin{array}{c}
s+n-3 \\
s
\end{array}\right),
$$

where we set $l=k+j$. To calculate the eigenvalues of the square of the higher spin Dirac operator $D_{j}^{2}$, we need the following lemma.

Lemma 3.3 On $V_{j}(k, s)^{\prime}$, it holds that

$$
\left.\left(\Delta_{j}-\left(s(s+n-2)-\frac{n(n-1)}{8}\right)\right)\right|_{V_{j}(k, s)^{\prime}}=\left(j+k+\frac{n}{2}\right)^{2} .
$$

Proof The standard Laplacian $\Delta_{j}$ on $S_{j}$ coincides with the Casimir operator $c_{2} / 2$ for $\operatorname{Spin}(n+1)$. By Freudenthal's formula, the eigenvalue of the $c_{2} / 2$ is $\pi_{\rho}\left(c_{2} / 2\right)=\langle\rho, \rho\rangle+2\left\langle\rho, \delta_{\operatorname{Spin}(n+1)}\right\rangle$. The inner product is given by $\langle\mu, v\rangle=\sum_{1 \leq i \leq m} \mu^{i} v^{i}$, and $\delta_{\mathrm{Spin}(n+1)}$ is half the sum of the positive roots,

$$
\delta_{\operatorname{Spin}(n+1)}= \begin{cases}(m-1 / 2, m-3 / 2, \cdots, 3 / 2,1 / 2) & \text { for } n+1=2 m+1, \\ (m-1, m-2, \cdots, 1,0) & \text { for } n+1=2 m .\end{cases}
$$

Then,

$$
\begin{aligned}
& \pi_{\rho}\left(c_{2} / 2\right)-\left(s(s+n-2)-\frac{n(n-1)}{8}\right) \\
= & (k+j+1 / 2)(k+j+n-1 / 2)+(s+1 / 2)(s+n-5 / 2) \\
& \quad+(n-4)(n-3) / 8-\left(s(s+n-2)-\frac{n(n-1)}{8}\right) \\
= & (j+k+n / 2)^{2} .
\end{aligned}
$$

From the factorization formula in Theorem 2.9, we can guess the eigenvalue $\lambda^{2}$ of $D_{j}^{2}$ on $V_{j}(k, s)^{\prime}$ is $\frac{(n+2 s-2)^{2}}{(n+2 j-2)^{2}}\left(j+k+\frac{n}{2}\right)^{2}$. In fact, we have 
Theorem 3.4 On the $n$-dimensional standard sphere $S^{n}$, the eigenvalue of $D_{j}^{2}$ on $V_{j}(k, s)^{\prime}$ is

$$
\frac{(n+2 s-2)^{2}}{(n+2 j-2)^{2}}\left(j+k+\frac{n}{2}\right)^{2}
$$

with multiplicity $\operatorname{dim} V_{j}(k, s)^{\prime}$.

Proof We shall prove the case of $n=2 m-1$ by induction for $j \geq 0$. First, we consider the case of $j=0$. It is well known that the spectrum of the Dirac operator $D_{0}^{2}$ on the sphere is $(k+n / 2)^{2}$ on $V_{0}(k, 0)^{\prime}$ for $k=0,1,2, \ldots$. Next, assuming the statement in the theorem for $j$ holds, we prove the one for $j+1$. We take $\phi$ in $V_{j}(k, s)^{\prime}$. It follows from our assumption, Lemma 3.3 and (11) that

$$
\left(T_{j}^{+}\right)^{*} T_{j}^{+} \phi=\frac{k(j-s+1)(2 j+k+n)(j+s+n-1)}{(j+n / 2)^{2}} \phi .
$$

Then,

$$
\operatorname{ker} T_{j}^{+}=\bigoplus_{0 \leq s \leq j} V_{j}(0, s)^{\prime},
$$

and $T_{j}^{+}: V_{j}(k, s)^{\prime} \rightarrow V_{j+1}(k-1, s)^{\prime}$ for any $k \geq 1$ is an isomorphism as $\operatorname{Spin}(n+1)$-module. Here, we use Schur's lemma with respect to the $\operatorname{Spin}(n+1)$-invariant operator $T_{j}^{+}$. Taking such a $T_{j}^{+} \phi$ in $V_{j+1}(k-1, s)^{\prime}$, we show

$$
D_{j+1}^{2} T_{j}^{+} \phi=\frac{(n+2 j-2)^{2}}{(n+2 j)^{2}} T_{j}^{+} D_{j}^{2} \phi=\frac{(n+2 s-2)^{2}}{(n+2 j)^{2}}\left(j+1+(k-1)+\frac{n}{2}\right)^{2} T_{j}^{+} \phi .
$$

The case of $s=j+1$ remains to be proved. According to Proposition $2.1, L^{2}\left(S_{j+1}\right)$ decomposes as $\operatorname{ker} T_{j+1}^{-} \oplus$ Image $T_{j}^{+}$. Then,

$$
\operatorname{ker} T_{j+1}^{-}=\bigoplus_{k \geq 0} V_{j+1}(k, j+1)^{\prime} .
$$

When $\phi$ is in $V_{j+1}(k, j+1)^{\prime}$, Weitzenböck formula (11) gives

$$
D_{j+1}^{2} \phi=\Delta_{j+1} \phi-\left((j+1)(n+j-1)-\frac{n(n-1)}{8}\right) \phi=\left((j+1)+k+\frac{n}{2}\right)^{2} \phi .
$$

Thus, we have proved the statement for $j+1$. We can prove the case of $n=2 m$ when we double each component $V_{j}(k, s)^{\prime}$ in the above proof.

By using Corollary 2.8, we calculate all the eigenvalues of the other generalized gradients on $S_{j}$.

Corollary 3.5 The eigenvalues of $\left(T_{j}^{+}\right)^{*} T_{j}^{+},\left(T_{j}^{-}\right)^{*} T_{j}^{-}$and $U_{j}^{*} U_{j}$ on $V_{j}(k, s)^{\prime}$ are given by

$$
\frac{k(j-s+1)(2 j+k+n)(j+s+n-1)}{(j+n / 2)^{2}},
$$




$$
\begin{aligned}
& \frac{(k+1)(j-s)(2 j+k+n-1)(j+s+n-2)}{(j+n / 2-1)^{2}}, \\
& \text { and } \frac{(n-3) s(j+k+1)(n+s-2)(j+k+n-1)}{(n-2)(j+1)(j+n-2)},
\end{aligned}
$$

respectively.

We investigate how the eigenspaces $\left\{V_{j}(k, s)^{\prime}\right\}_{k, j, s}$ relate to each other through the generalized gradients. Put

$$
\mathbf{V}_{j}(s)^{\prime}:=\bigoplus_{k \geq 0} V_{j}(k, s)^{\prime}
$$

for each $j$ and $s=0, \cdots, j$, and we show that $\mathbf{V}_{j}(s)^{\prime}$ is just $W_{s}$ in Theorem 2.11. Remark that, in the case of the sphere, $W_{s}$ coincides with the kernel of $B(s ; j)$. The following diagram is useful to understand relations between the spaces:

$$
\begin{aligned}
& L^{2}\left(S_{0}\right)=\mathbf{V}_{0}(0)^{\prime} \\
& \Downarrow T_{0}^{+} \\
& L^{2}\left(S_{j-1}\right)=\mathbf{V}_{j-1}(0)^{\prime} \oplus \cdots \oplus \quad \mathbf{V}_{j-1}(j-1)^{\prime} \oplus \quad\{0\} \\
& \left.T_{j}^{-}\right\rfloor \Downarrow T_{j-1}^{+} \quad T_{j}^{-} \bigcup_{\Downarrow} T_{j-1}^{+} \quad T_{j}^{-} \uparrow
\end{aligned}
$$

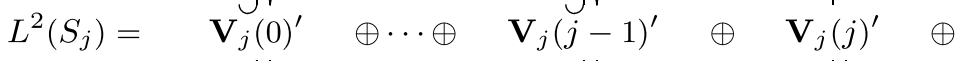

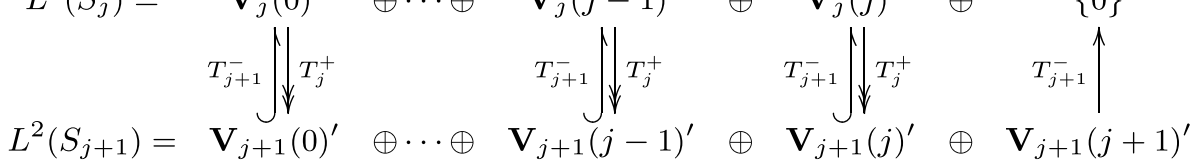

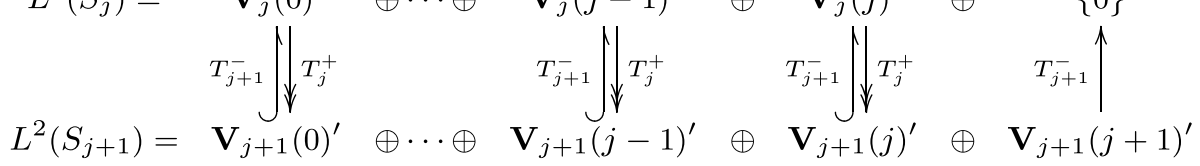

Here, we change each $\mathbf{V}_{j}(s)^{\prime}$ by $2 \mathbf{V}_{j}(s)^{\prime}$ when $n$ is $2 m$. Then, we have the proposition.

Proposition 3.6 The kernels and images of the generalized gradients are realized as $\operatorname{Spin}(n+1)$-module in $L^{2}\left(S^{n}, S_{j}\right)$ for $j=0,1, \cdots$ as follows.

For $n=2 m-1$,

$$
\begin{gathered}
\operatorname{ker} T_{j}^{+} \cong \bigoplus_{0 \leq s \leq j} V_{j}(0, s)^{\prime}, \quad \operatorname{ker} T_{j}^{-} \cong \mathbf{V}_{j}(j)^{\prime}, \quad \operatorname{ker} U_{j} \cong \mathbf{V}_{j}(0)^{\prime}, \\
\text { Image } T_{j-1}^{+} \cong \bigoplus_{0 \leq s \leq j-1} \mathbf{V}_{j}(s)^{\prime}, \quad \text { Image } T_{j+1}^{-} \cong L^{2}\left(S^{n}, S_{j}\right) \ominus \operatorname{ker} T_{j}^{+} .
\end{gathered}
$$

In particular, we have

$$
\operatorname{ker} T_{j}^{+} \cap \operatorname{ker} T_{j}^{-} \cong V_{j}(0, j)^{\prime}, \quad \operatorname{ker} T_{j}^{+} \cap \operatorname{ker} U_{j} \cong V_{j}(0,0)^{\prime} .
$$

For $n=2 m$, we double each component $V_{j}(k, s)^{\prime}$ and $\mathbf{V}_{j}(s)^{\prime}$ on the above equations. 


\section{Generalized gradients on $j$-th symmetric tensor fields}

In this section, we study the generalized gradients on the bundle of trace-free symmetric tensors $\operatorname{Sym}_{0}^{j}=\operatorname{Sym}_{0}^{j}\left(T M^{c}\right)$. Let $(M, g)$ be an oriented Riemannian manifold. The bundle $\operatorname{Sym}^{j}\left(T M^{c}\right)$ for $j$-th symmetric tensor fields splits as (2) and the primitive component $\mathrm{Sym}_{0}^{j}$ is a vector bundle associated with the (oriented) orthonormal frame bundle $\mathrm{SO}(M)$ with the highest weight $\left(j, 0_{m-1}\right)$. We compose the covariant derivative $\nabla$ and the orthogonal projection along the decomposition

$$
\operatorname{Sym}_{0}^{j} \otimes T M^{c}=\operatorname{Sym}_{0}^{j, 1} \oplus \operatorname{Sym}_{0}^{j+1} \oplus \operatorname{Sym}_{0}^{j-1},
$$

where $\operatorname{Sym}_{0}^{j, 1}$ is an irreducible vector bundle with the highest weight $\left(j, 1,0_{m-2}\right)$. Then, we have three generalized gradients

$$
\begin{aligned}
T_{j}^{+}: \Gamma\left(\operatorname{Sym}_{0}^{j}\right) \rightarrow \Gamma\left(\operatorname{Sym}_{0}^{j+1}\right), \quad U_{j}: \Gamma\left(\operatorname{Sym}_{0}^{j}\right) \rightarrow \Gamma\left(\operatorname{Sym}_{0}^{j, 1}\right), \\
T_{j}^{-}: \Gamma\left(\operatorname{Sym}_{0}^{j}\right) \rightarrow \Gamma\left(\operatorname{Sym}_{0}^{j-1}\right),
\end{aligned}
$$

where we set $U_{0}=0$ and $T_{0}^{-}=0$.

Remark 4.1 For $n=4, \mathrm{Sym}_{0}^{j .1}$ splits into the sum of SO(4)-modules with the highest weight $(j, 1)$ and $(j,-1)$. For $n=3$ and $j \geq 1, \operatorname{Sym}_{0}^{j}$ appears again instead of $\operatorname{Sym}_{0}^{j, 1}$. We denote by $U_{j}$ the self-gradient from $\Gamma\left(\mathrm{Sym}_{0}^{j}\right)$ to $\Gamma\left(\mathrm{Sym}_{0}^{j}\right)$ on a 3-dim Riemannian manifold, which is not an elliptic operator.

It follows from Weitzenböck formulas in [16] that there are two identities among them

$$
\begin{aligned}
\nabla^{*} \nabla & =\left(T_{j}^{+}\right)^{*} T_{j}^{+}+U_{j}^{*} U_{j}+\left(T_{j}^{-}\right)^{*} T_{j}^{-}, \\
\frac{1}{2} R_{\mathrm{Sym}_{0}^{j}} & =-j\left(T_{j}^{+}\right)^{*} T_{j}^{+}+U_{j}^{*} U_{j}+(n+j-2)\left(T_{j}^{-}\right)^{*} T_{j}^{-} .
\end{aligned}
$$

Eliminating $U_{j}$ from the above equations, we find

$$
\begin{aligned}
\Delta_{j}: & =\nabla^{*} \nabla+\frac{1}{2} R_{\mathrm{Sym}_{0}^{j}}=\nabla^{*} \nabla-\frac{1}{2} R_{\mathrm{Sym}_{0}^{j}}+R_{\mathrm{Sym}_{0}^{j}} \\
& =(j+1)\left(T_{j}^{+}\right)^{*} T_{j}^{+}-(n+j-3)\left(T_{j}^{-}\right)^{*} T_{j}^{-}+R_{\mathrm{Sym}_{0}^{j}} .
\end{aligned}
$$

As in the case of the higher spin fields, we have to compare $T_{j+1}^{-}$and $\left(T_{j}^{+}\right)^{*}$ from $\Gamma\left(\operatorname{Sym}_{0}^{j+1}\right)$ to $\Gamma\left(\operatorname{Sym}_{0}^{j}\right)$. The next lemma follows from [16].

Lemma 4.2 Let $D_{\lambda}^{\rho}=\Pi_{\lambda} \circ \nabla: \Gamma\left(S_{\rho}\right) \rightarrow \Gamma\left(S_{\lambda}\right)$ be a generalized gradient on an irreducible vector bundle $S_{\rho}=\mathrm{SO}(M) \times_{S O(n)} W_{\rho}$ over a Riemannian manifold $(M, g)$. Here, $\Pi_{\lambda}$ is the orthogonal projection onto $S_{\lambda}$ from $S_{\rho} \otimes T M^{c}$. Then,

$$
\left(D_{\lambda}^{\rho}\right)^{*} D_{\lambda}^{\rho}=\frac{\operatorname{dim} W_{\lambda}}{\operatorname{dim} W_{\rho}} D_{\rho}^{\lambda}\left(D_{\rho}^{\lambda}\right)^{*} .
$$

Proof The principal symbol of $D_{\lambda}^{\rho}$ is given by the linear map $p_{\lambda}^{\rho}(\xi)$ from $W_{\rho}$ to $W_{\lambda}$ defined by 


$$
p_{\lambda}^{\rho}(\xi) \phi:=\Pi_{\lambda}(\phi \otimes \xi), \quad \text { for } \phi \text { in } W_{\rho} \text { and } \xi \text { in } \mathbb{C}^{n}=T_{x}^{*} M^{c} .
$$

In other words, $D_{\lambda}^{\rho}$ is $\sum_{i} p_{\lambda}^{\rho}\left(e_{i}\right) \nabla_{e_{i}}$ as the Dirac operator $D$ is given by $\sum_{i} e_{i} \cdot \nabla_{e_{i}}$. We denote by $p_{\lambda}^{\rho}(\xi)^{*}$ its adjoint map from $W_{\lambda}$ to $W_{\rho}$. Conversely we know that the orthogonal projection $\Pi_{\lambda}$ is realized by using the principal symbol (see Lemma 4.13 in [16]),

$$
\Pi_{\lambda}(\phi \otimes \xi)=\sum_{1 \leq i \leq n}\left(p_{\lambda}^{\rho}\left(e_{i}\right)^{*} p_{\lambda}^{\rho}(\xi) \phi\right) \otimes e_{i}
$$

where $\left\{e_{i}\right\}_{i}$ is an orthonormal basis for $\mathbb{C}^{n}$. We shall calculate the following two $\operatorname{SO}(n)$ -invariant maps from $W_{\rho}$ to $W_{\rho}$, which are constants by Schur's lemma,

$$
\sum_{i} p_{\lambda}^{\rho}\left(e_{i}\right)^{*} p_{\lambda}^{\rho}\left(e_{i}\right), \quad \sum_{i} p_{\rho}^{\lambda}\left(e_{i}\right) p_{\rho}^{\lambda}\left(e_{i}\right)^{*}
$$

Let $\left\{\phi_{\alpha}\right\}_{\alpha}$ be a basis of $W_{\rho}$. We consider the trace of $\Pi_{\lambda}: W_{\rho} \otimes \mathbb{C}^{n} \rightarrow W_{\lambda}$.

$$
\begin{aligned}
\operatorname{dim} W_{\lambda} & =\sum_{\alpha, i}\left\langle\Pi_{\lambda}\left(\phi_{\alpha} \otimes e_{i}\right), \phi_{\alpha} \otimes e_{i}\right\rangle=\sum_{\alpha, i, j}\left\langle\left(p_{\lambda}^{\rho}\left(e_{j}\right)^{*} p_{\lambda}^{\rho}\left(e_{i}\right) \phi_{\alpha}\right) \otimes e_{j}, \phi_{\alpha} \otimes e_{i}\right\rangle \\
& =\sum_{\alpha, i}\left\langle p_{\lambda}^{\rho}\left(e_{i}\right)^{*} p_{\lambda}^{\rho}\left(e_{i}\right) \phi_{\alpha}, \phi_{\alpha}\right\rangle=\sum_{i} p_{\lambda}^{\rho}\left(e_{i}\right)^{*} p_{\lambda}^{\rho}\left(e_{i}\right) \sum_{\alpha}\left\langle\phi_{\alpha}, \phi_{\alpha}\right\rangle \\
& =\operatorname{dim} W_{\rho} \sum_{i} p_{\lambda}^{\rho}\left(e_{i}\right)^{*} p_{\lambda}^{\rho}\left(e_{i}\right) .
\end{aligned}
$$

Then, we have $\sum_{i} p_{\lambda}^{\rho}\left(e_{i}\right)^{*} p_{\lambda}^{\rho}\left(e_{i}\right)=\frac{\operatorname{dim} W_{\lambda}}{\operatorname{dim} W_{\rho}} \operatorname{id}_{W_{\rho}}$. On the other hand, we have

$$
\begin{aligned}
\left\langle p_{\rho}^{\lambda}(\xi) \phi, p_{\rho}^{\lambda}(\eta) \psi\right\rangle & =\left\langle\Pi_{\rho}(\phi \otimes \xi), \Pi_{\rho}(\psi \otimes \eta)\right\rangle \\
& =\sum_{i, j}\left\langle p_{\rho}^{\lambda}\left(e_{i}\right)^{*} p_{\rho}^{\lambda}(\xi) \phi \otimes e_{i}, p_{\rho}^{\lambda}\left(e_{j}\right)^{*} p_{\rho}^{\lambda}(\eta) \psi \otimes e_{j}\right\rangle \\
& =\sum_{i}\left\langle p_{\rho}^{\lambda}\left(e_{i}\right)^{*} p_{\rho}^{\lambda}(\xi) \phi, p_{\rho}^{\lambda}\left(e_{i}\right)^{*} p_{\rho}^{\lambda}(\eta) \psi\right\rangle \\
& =\sum_{i}\left\langle p_{\rho}^{\lambda}\left(e_{i}\right) p_{\rho}^{\lambda}\left(e_{i}\right)^{*} p_{\rho}^{\lambda}(\xi) \phi, p_{\rho}^{\lambda}(\eta) \psi\right\rangle \\
& =\left(\sum_{i} p_{\rho}^{\lambda}\left(e_{i}\right) p_{\rho}^{\lambda}\left(e_{i}\right)^{*}\right)\left\langle p_{\rho}^{\lambda}(\xi) \phi, p_{\rho}^{\lambda}(\eta) \psi\right\rangle .
\end{aligned}
$$

Then, we have $\sum_{i} p_{\rho}^{\lambda}\left(e_{i}\right) p_{\rho}^{\lambda}\left(e_{i}\right)^{*}=\mathrm{id}_{W_{\rho}}$. Since there is a nonzero constant $a$ such that $p_{\lambda}^{\rho}(\xi)=a p_{\rho^{\lambda}}^{\lambda}(\xi)^{*}$, we have concluded that $|a|^{2}=\operatorname{dim} W_{\lambda} / \operatorname{dim} W_{\rho}$ and hence $\left(D_{\lambda}^{\rho}\right)^{*} D_{\lambda}^{\rho}=\frac{\rho_{\operatorname{dim} W_{\lambda}}}{\operatorname{dim} W_{\rho}} D_{\rho}^{\lambda}\left(D_{\rho}^{\lambda}\right)^{*}$.

Thus, we have given relations among the generalized gradients $\left\{T_{j}^{ \pm}\right\}_{j}$ to construct a factorization formula on a space of constant curvature.

Proposition 4.3 On a space $(M, g)$ of constant curvature $K=c$, the operators $T_{j}^{+}, T_{j}^{-}$and $\Delta_{j}$ for $j=0,1,2, \ldots$ satisfy 


$$
\begin{aligned}
\Delta_{j} & =(j+1)\left(T_{j}^{+}\right)^{*} T_{j}^{+}-(n+j-3)\left(T_{j}^{-}\right)^{*} T_{j}^{-}+2 j(n+j-2) c, \\
\frac{1}{2} R_{\mathrm{Sym}_{0}^{j}} & =j(n+j-2) c=-j\left(T_{j}^{+}\right)^{*} T_{j}^{+}+U_{j}^{*} U_{j}^{+}+(n+j-2)\left(T_{j}^{-}\right)^{*} T_{j}^{-}, \\
\left(T_{j+1}^{-}\right)^{*} T_{j+1}^{-} & =\frac{(j+1)(n+2 j-2)}{(n+j-2)(n+2 j)} T_{j}^{+}\left(T_{j}^{+}\right)^{*} .
\end{aligned}
$$

Remark 4.4 We do not normalize constant multiples of the operators $\left\{T_{j}^{ \pm}\right\}_{j}$ as the previous section. When we use a notation in [14], we find

$$
\left(T_{j}^{+}\right)^{*} T_{j}^{+}=\frac{1}{j+1} d_{0}^{*} d_{0}, \quad\left(T_{j}^{-}\right)^{*} T_{j}^{-}=\frac{n+2 j-4}{(n+2 j-2)(n+j-3)} \delta^{*} \delta .
$$

We show the factorization formula for the $j+1$-st power of the Laplacian on $j$-th symmetric tensor fields, which will be useful to calculate the eigenvalues on the sphere in the next section.

Theorem 4.5 (Factorization formula) Let $(M, g)$ be a space of constant curvature $K=c$, and $\mathrm{Sym}_{0}^{j}$ the vector bundle of trace-free symmetric tensor fields on $(M, g)$. The opera$\operatorname{tor} T_{j}^{+}: \Gamma\left(\operatorname{Sym}_{0}^{j}\right) \rightarrow \Gamma\left(\operatorname{Sym}_{0}^{j+1}\right)$ and the Lichnerowicz Laplacian $\Delta_{j}: \Gamma\left(\operatorname{Sym}_{0}^{j}\right) \rightarrow \Gamma\left(\operatorname{Sym}_{0}^{j}\right)$ satisfy

$$
\prod_{s=0}^{j}\left(\left(T_{j}^{+}\right)^{*} T_{j}^{+}-a(s ; j)\left(\Delta_{j}-b(s ; j) c\right)\right)=0,
$$

where $\quad a(s ; j)=\frac{(j-s+1)(n+j+s-2)}{(j+1)(n+2 j-2)}, \quad b(s ; j)=j(n+j-1)+s(n+s-3)$.

Proof We prove the theorem by induction for $j$. The case of $j=0$ follows from the definition of $\Delta_{0}$, that is, $\Delta_{0}=\left(T_{0}^{+}\right)^{*} T_{0}^{+}$. We assume that the equation (20) holds for $j$. By (19),

$$
\begin{aligned}
& T_{j}^{+}\left(\left(T_{j}^{+}\right)^{*} T_{j}^{+}-a(s ; j)\left(\Delta_{j}-b(s ; j) c\right)\right) \\
= & \left(T_{j}^{+}\left(T_{j}^{+}\right)^{*}-a(s ; j)\left(\Delta_{j+1}-b(s ; j) c\right)\right) T_{j}^{+} \\
= & \underbrace{\frac{(j+2)(n+2 j)}{(j+1)(n+2 j-2)}}_{=: k(j)}\left(\left(T_{j+1}^{+}\right)^{*} T_{j+1}^{+}-a(s ; j+1)\left(\Delta_{j+1}-b(s ; j+1) c\right)\right) T_{j}^{+} .
\end{aligned}
$$

Then, 


$$
\begin{aligned}
0=T_{j}^{+}\left\{\prod_{s=0}^{j}\left(\left(T_{j}^{+}\right)^{*} T_{j}^{+}-a(s ; j)\left(\Delta_{j}-b(s ; j) c\right)\right)\right\}\left(T_{j}^{+}\right)^{*} \\
=k(j)^{j}\left\{\prod_{s=0}^{j}\left(\left(T_{j+1}^{+}\right)^{*} T_{j+1}^{+}-a(s ; j+1)\left(\Delta_{j+1}-b(s ; j+1) c\right)\right)\right\} T_{j}^{+}\left(T_{j}^{+}\right)^{*} \\
=k(j)^{j+1}\left\{\prod_{s=0}^{j}\left(\left(T_{j+1}^{+}\right)^{*} T_{j+1}^{+}-a(s ; j+1)\left(\Delta_{j+1}-b(s ; j+1) c\right)\right)\right\} \\
\times\left(\left(T_{j+1}^{+}\right)^{*} T_{j+1}^{+}-a(j+1 ; j+1)\left(\Delta_{j+1}-b(j+1 ; j+1) c\right)\right) .
\end{aligned}
$$

Finally we reach (20) for $j+1$.

If a tensor field $\phi$ in $\Gamma\left(\operatorname{Sym}_{0}^{j}\right)$ satisfies $T_{j}^{+} \phi=0$, then we call it trace-free conformal Killing tensor, which has been investigated not only in differential geometry but also in relativity theory. As a corollary, we have an information of the eigenvalues of the Laplacian on trace-free conformal Killing tensors on a compact space of constant curvature $([25,26]$ for the sphere case). We introduce a filtration on $\Gamma\left(\mathrm{Sym}_{0}^{j}\right)$ as

$$
F_{j} \subset F_{j+1} \subset \cdots \subset F_{0}=\Gamma\left(\operatorname{Sym}_{0}^{j}\right), \quad F_{k}=\operatorname{ker} T_{k}^{-} \cdots T_{j}^{-} .
$$

and an associated grading

$$
\Gamma\left(\operatorname{Sym}_{0}^{j}\right)=\bigoplus_{0 \leq s \leq j} W_{s}, \quad W_{s}:=T_{j-1}^{+} \cdots T_{s}^{+}\left(\operatorname{ker} T_{s}^{-}\right) .
$$

Since the inclusion

$$
W_{s} \subset \operatorname{ker}\left(\left(T_{j}^{+}\right)^{*} T_{j}^{+}-a(s ; j)\left(\Delta_{j}-b(s ; j) c\right)\right)
$$

follows from the same as in Theorem 2.11, we obtain the following corollary.

Corollary 4.6 On a compact space of constant curvature $K=c$, the eigenvalue of $\Delta_{j}$ on $\operatorname{ker} T_{j}^{+} \cap W_{s} i s b(s ; j)=(j(n+j-1)+s(n+s-3)) c$.

\section{Harmonic analysis for trace-free symmetric tensor fields on $S^{n}$}

The method to calculate the eigenvalues of the operators $\left\{\left(T_{j}^{ \pm}\right)^{*} T_{j}^{ \pm}, U_{j}^{*} U_{j}, \Delta_{j}\right\}_{j}$ on the $n$-dimensional sphere $S^{n}=\mathrm{SO}(n+1) / \mathrm{SO}(n)$ is the same as in the spinor case. From Frobenius reciprocity and the branching rule, the space of the trace-free symmetric tensor fields are decomposed with respect to $\mathrm{SO}(n+1)$,

$$
L^{2}\left(S^{n}, \operatorname{Sym}_{0}^{j}\right) \cong \bigoplus_{0 \leq s \leq j} \mathbf{V}_{j}(s)=\bigoplus_{0 \leq s \leq j}\left(\bigoplus_{k \geq 0} V_{j}(k, s)\right) .
$$

As mentioned in Sect. 3, the space $V_{j}(k, s)$ stands for an irreducible $\mathrm{SO}(n+1)$-module with the highest weight $\left(k+j, s, 0_{m-2}\right)$ and its dimension is 


$$
\frac{(2 l+n-1)(2 s+n-3)(l+n-2+s)(l+1-s)}{(n-1)(n-2)(s+n-3)(l+n-2)}\left(\begin{array}{c}
l+n-2 \\
l+1
\end{array}\right)\left(\begin{array}{c}
s+n-3 \\
s
\end{array}\right)
$$

by setting $l=k+j$. Note that, for $n=3, V_{j}(k, s)$ is the sum of $\operatorname{SO}(4)$-modules with the highest weight $(k+j, s)$ and $(k+j,-s)$. We also put $\mathbf{V}_{j}(s):=\bigoplus_{k \geq 0} V_{j}(k, s)$. As in [3] Freudenthal's formula gives the eigenvalue of $\Delta_{j}$ on $V_{j}(k, s)$,

$$
\left.\Delta_{j}\right|_{V_{j}(k, s)}=(j+k)(n+k+j-1)+s(s+n-3),
$$

so that we have

$$
\left.\left(\Delta_{j}-b(s ; j)\right)\right|_{V_{j}(k, s)}=k(n+k+2 j-1) .
$$

The factorization formula (20) allows us to guess that the eigenvalue of $\left(T_{j}^{+}\right)^{*} T_{j}^{+}$on $V_{j}(k, s)$ is

$$
a(s ; j) k(n+k+2 j-1)=\frac{k(n+k+2 j-1)(j-s+1)(n+j+s-2)}{(j+1)(n+2 j-2)} .
$$

We shall prove the claim that the eigenvalue of $\left(T_{j}^{+}\right)^{*} T_{j}^{+}$on $V_{j}(k, s)$ coincides with (21) by induction for $j$. When $j=0$, the claim is true because of $\Delta_{0}=\left(T_{0}^{+}\right)^{*} T_{0}^{+}$. We suppose the claim holds for $j$. Then,

$$
\operatorname{ker} T_{j}^{+} \cong \bigoplus_{0 \leq s \leq j} V_{j}(0, s)
$$

and $T_{j}^{+}: V_{j}(k, s) \rightarrow V_{j+1}(k-1, s)$ is an isomorphism for any $k \geq 1$ and $s=0, \cdots, j$. For $\phi$ in $V_{j}(k, s)$,

$$
\begin{aligned}
& (j+2)\left(T_{j+1}^{+}\right)^{*} T_{j+1}^{+}\left(T_{j}^{+} \phi\right) \\
= & \left(\Delta_{j+1}+(n+j-2)\left(T_{j+1}^{-}\right)^{*} T_{j+1}^{-}-2((j+1)(n+(j+1)-2))\right)\left(T_{j}^{+} \phi\right) \\
= & \left(\Delta_{j+1}+\frac{(j+1)(n+2 j-2)}{n+2 j} T_{j}^{+}\left(T_{j}^{+}\right)^{*}-2((j+1)(n+j-1))\right)\left(T_{j}^{+} \phi\right) \\
= & T_{j}^{+} \Delta_{j} \phi+\frac{k(n+k+2 j-1)(j-s+1)(n+j+s-2)}{n+2 j} T_{j}^{+} \phi \\
& -2((j+1)(n+j-1))\left(T_{j}^{+} \phi\right) \\
= & \frac{(k-1)(n+k+2 j)(j-s+2)(n+j+s-1)}{n+2 j} T_{j}^{+} \phi .
\end{aligned}
$$

Thus, the eigenvalues of $\left(T_{j+1}^{+}\right)^{*} T_{j+1}^{+}$on $V_{j+1}(k-1, s)$ for any $k \geq 1$ and $s=0, \cdots, j$ coincide with (21). The cases of $V_{j+1}(k, j+1)$ for any $k \geq 0$ remain to be proved. Since $T_{j}^{+}$is an overdetermined elliptic operator, the space of the sections $\Gamma\left(\operatorname{Sym}_{0}^{j+1}\right)$ decomposes into the orthogonal direct sum of $\operatorname{ker} T_{j+1}^{-}$and Image $T_{j}^{+}$. From the above discussion, it holds that Image $T_{j}^{+}$is given by $\bigoplus_{0 \leq s \leq j} \mathbf{V}_{j+1}(s)$. Then, its orthogonal complement $\operatorname{ker} T_{j+1}^{-}$is $\mathbf{V}_{j+1}(j+1)=\bigoplus_{k \geq 0} V_{j+1}(k, j+1)$. For $\phi$ in $V_{j+1}(k, j+1)$, by Weitzenböck formula, we have 


$$
\left(T_{j+1}^{+}\right)^{*} T_{j+1}^{+} \phi=\frac{1}{j+2}\left(\Delta_{j+1} \phi-2((j+1)(n+(j+1)-2)) \phi\right)=\frac{k(n+k+2 j+1)}{j+2} \phi,
$$

and check that this gives $(21)$ on $V_{j+1}(k, j+1)$. Thus, we have proved that $(21)$ gives the eigenvalue of $\left(T_{j}^{+}\right)^{*} T_{j}^{+}$on $V_{j}(k, s)$ for any $j$. We can also have the eigenvalue of the other operators by using Weitzenböck formulas in Proposition 4.3.

Theorem 5.1 On the $n$-dimensional standard sphere $S^{n}$, the eigenvalues of $\left(T_{j}^{+}\right)^{*} T_{j}^{+}$, $\left(T_{j}^{-}\right)^{*} T_{j}^{-}, U_{j}^{*} U_{j}$ and $\Delta_{j}$ on $V_{j}(k, s)$ are given by

$$
\begin{gathered}
\frac{k(n+k+2 j-1)(j-s+1)(n+j+s-2)}{(j+1)(n+2 j-2)}, \\
\frac{(j-s)(k+1)(n+k+2 j-2)(n+j+s-3)}{(n+j-3)(n+2 j-2)}, \\
\frac{s(k+j+1)(n+s-3)(n+k+j-2)}{(j+1)(n+j-3)}, \\
\text { and } \quad(k+j)(k+j+n-1)+s(n+s-3),
\end{gathered}
$$

respectively. The kernels and images of the operators are realized as $\mathrm{SO}(n+1)$-modules in $L^{2}\left(S^{n}, \operatorname{Sym}_{0}^{j}\right)$ as follows:

$$
\begin{array}{r}
\operatorname{ker} T_{j}^{+} \cong \bigoplus_{0 \leq s \leq j} V_{j}(0, s), \quad \operatorname{ker} T_{j}^{-} \cong \mathbf{V}_{j}(j), \quad \operatorname{ker} U_{j}^{-} \cong \mathbf{V}_{j}(0), \\
\text { Image } T_{j-1}^{+} \cong \bigoplus_{0 \leq s \leq j-1} \mathbf{V}_{j}(s), \quad \text { Image } T_{j+1}^{-} \cong L^{2}\left(S^{n}, \operatorname{Sym}_{0}^{j}\right) \ominus \operatorname{ker} T_{j}^{+} .
\end{array}
$$

We also know that

$$
\operatorname{ker} T_{j}^{+} \cap \operatorname{ker} T_{j}^{-} \cong V_{j}(0, j), \quad \operatorname{ker} T_{j}^{+} \cap \operatorname{ker} U_{j} \cong V_{j}(0,0) .
$$

As an interesting application to geometry, we shall discuss the space of Killing tensor fields on $S^{n}$ from the viewpoint of representation theory. We consider the differential $d$ on the symmetric tensor fields defined by

$$
d: \Gamma\left(\mathrm{Sym}^{j}\right) \ni K \mapsto d K:=\sum_{i=1}^{n} e_{i} \cdot \nabla_{e_{i}} K \in \Gamma\left(\mathrm{Sym}^{j+1}\right),
$$

where $\left\{e_{i}\right\}_{i}$ is a local orthonormal frame for $T M$ and $e_{i} \cdot$ denotes the symmetric tensor product by $e_{i}$. If $d K$ is zero, then $K$ is said to be a Killing tensor field. Since Killing tensor fields give the first integrals for geodesics, they play an important role in the physics literature, especially in relativity theory. We refer to [14] for general results of Killing and conformal Killing tensor fields in Riemannian geometry. Since the differential $d$ is a derivation on $\oplus_{j} \Gamma\left(\mathrm{Sym}^{j}\right)$, that is,

$$
d\left(K \cdot K^{\prime}\right)=(d K) \cdot K^{\prime}+K \cdot\left(d K^{\prime}\right) \quad \text { for } K, K^{\prime} \text { in } \oplus_{j} \Gamma\left(\mathrm{Sym}^{j}\right)
$$


the space $K(M)$ of the Killing tensor fields is a graded algebra, $K(M)=\bigoplus_{j} K^{j}(M)$, where $K^{j}(M)$ is the space of the Killing tensor fields with degree $j$. Let $K$ be a symmetric tensor field with degree $j$. This $K$ is decomposed as

$$
K=K_{0}+g \cdot K_{1}+g^{2} \cdot K_{2}+\cdots+g^{l} \cdot K_{l} \quad\left(K_{i} \in \Gamma\left(\operatorname{Sym}_{0}^{j-2 i}\right), \quad 0 \leq i \leq l=[j / 2]\right)
$$

with respect to (2), where $K_{0}$ is the trace-free part of $K$. Since the derivative $d$ on $\Gamma\left(\operatorname{Sym}_{0}^{j-2 i}\right)$ is realized as a linear combination of the generalized gradients $T_{j-2 i}^{+}$and $T_{j-2 i}^{-}$, the tensor field $d K_{i}$ has components only in $\Gamma\left(\operatorname{Sym}_{0}^{j-2 i+1}\right)$ and $g \cdot \Gamma\left(\operatorname{Sym}_{0}^{j-2 i-i}\right)$, and hence $d K_{i}=\left(d K_{i}\right)_{0}+g \cdot\left(d K_{i}\right)_{1}$. Then, it is easily shown that $K$ is a Killing tensor field if and only if $\left\{K_{i}\right\}_{0 \leq i \leq l}$ satisfy

$$
\left(d K_{0}\right)_{0}=0, \quad\left(d K_{0}\right)_{1}+g \cdot\left(d K_{1}\right)_{0}=0, \ldots,\left(d K_{l-1}\right)_{1}+g \cdot\left(d K_{l}\right)_{0}=0, \quad\left(d K_{l}\right)_{1}=0 .
$$

Note that if $K$ satisfies only the first condition $\left(d K_{0}\right)_{0}=0$, then $K$ is said to be a conformal Killing tensor field. For a trace-free symmetric tensor $K=K_{0}$ with degree $j$, $K$ is a trace-free Killing tensor (resp. trace-free conformal Killing tensor) if and only if $K$ is in $\operatorname{ker} T_{j}^{+} \cap \operatorname{ker} T_{j}^{-}$(resp. in $\operatorname{ker} T_{j}^{+}$). An important observation is that there is a nonzero constant $c=c(i, j)$ such that $\left(d K_{i}\right)_{1}+g \cdot\left(d K_{i+1}\right)_{0}=0$ can be rewritten as $T_{j-2 i}^{-}\left(K_{i}\right)=c T_{j-2 i-2}^{+}\left(K_{i+1}\right)$.

We consider Killing tensor fields on the standard sphere. Let $K=K_{0}+g \cdot K_{1}+\cdots$ be a primitive Killing tensor field with degree $j$, that is, $K$ is in $K^{j}\left(S^{n}\right)$ and orthogonal to $g \cdot K^{j-2}\left(S^{n}\right)$ with respect to the natural $L^{2}$-inner product on the space of the symmetric tensors $\Gamma\left(\mathrm{Sym}^{j}\right)$;

$$
K^{j}\left(S^{n}\right)=P^{j}\left(S^{n}\right) \oplus g \cdot K^{j-2}\left(S^{n}\right),
$$

where $P^{j}\left(S^{n}\right)$ is the space of the primitive Killing tensor fields with degree $j$. Then, we may assume $K_{0}$ is in $V_{j}(0, s)$ because of $T_{j}^{+}\left(K_{0}\right)=0$. When $s=j$, we know $T_{j}^{-}\left(K_{0}\right)$ is zero by Theorem 5.1 and hence $T_{j-2}^{+}\left(K_{1}\right)$ is zero by (26). Thus, we know $K$ is the form of $K=K_{0}+g \cdot \tilde{K}_{1}$ with $\tilde{K}_{1}$ in $K^{j-2}\left(S^{n}\right)$. Therefore, $K=K_{0}$ due to primitiveness of $K$. When $s=j-1$, we know that $T_{j}^{-}\left(K_{0}\right)=c T_{j-2}^{+}\left(K_{1}\right)$ is not zero and $K_{1}$ has to be in a $\mathrm{SO}(n+1)$ -module with the highest weight $\left(j, j-1,0_{m-2}\right)$ because $T_{j}^{ \pm}$is an invariant operator. Since $\Gamma\left(\operatorname{Sym}_{0}^{j-2}\right)$ does not include such a component, there is no Killing tensor field $K$ with $K_{0}$ in $V_{j}(0, j-1)$. In the same manner, when $K_{0}$ is in $V_{j}(0, j-2 i)$, the primitive Killing tensor $K$ is a form of $K=K_{0}+g \cdot K_{1}+\cdots+g^{i} \cdot K_{i}$ with $K_{k}$ in $V_{j-2 k}(2 k, j-2 i) \cong V_{j}(0, j-2 i)$ for $k=0, \cdots, i$, where each $K_{k}$ is determined by $K_{0}$ through (26). We also know there is no Killing tensor field $K$ with nonzero $K_{0}$ in $V_{j}(0, j-2 i+1)$.

Proposition 5.2 ([26]) Let $P^{j}\left(S^{n}\right)$ be the space of the primitive Killing tensors with degree $j$ on the standard sphere. Then,

$$
\begin{aligned}
& P^{j}\left(S^{n}\right) \cong \bigoplus_{0 \leq i \leq j j / 2]} V_{j}(0, j-2 i)=\bigoplus_{0 \leq i \leq[j / 2]}\left(j, j-2 i, 0_{m-2}\right), \\
& K^{j}\left(S^{n}\right)=\bigoplus_{0 \leq i \leq[j / 2]} g^{i} \cdot P^{j-2 i}\left(S^{n}\right) .
\end{aligned}
$$




\section{Higher spin Dirac operators on spinor fields with differential forms}

First we survey the analysis of generalized gradients on differential forms. Let $(M, g)$ be an oriented Riemannian manifold and $\Lambda^{j}\left(T^{*} M\right)$ be the bundle of differential forms. Due to the star operator $*: \Lambda^{j}\left(T^{*} M\right) \rightarrow \Lambda^{n-j}\left(T^{*} M\right)$, we have to study only the case of $j \leq[n / 2]$. As presented in $[16,24]$, there are three differential operators

$$
\begin{aligned}
& C: \Omega^{j}(M)=\Gamma\left(\Lambda^{j}\left(T^{*} M\right)\right) \rightarrow \Gamma\left(\Lambda^{j, 1}\left(T^{*} M\right)\right), \\
& d: \Omega^{j}(M) \rightarrow \Omega^{j+1}(M), \quad d^{*}: \Omega^{j}(M) \rightarrow \Omega^{j-1}(M)
\end{aligned}
$$

satisfying

$$
\begin{gathered}
\Delta_{j}=\nabla^{*} \nabla+\frac{1}{2} R_{\Lambda^{j}}=d^{*} d+d d^{*}, \quad d d=0, \quad d^{*} d^{*}=0, \\
\nabla^{*} \nabla=C^{*} C+\frac{1}{j+1} d^{*} d+\frac{1}{n-j+1} d d^{*},
\end{gathered}
$$

where the highest weight of the bundle $\Lambda^{j, 1}\left(T^{*} M\right)$ with respect to $\operatorname{SO}(n)$ is

$$
\begin{cases}\left(2,1_{j-1}, 0_{m-1-j}\right) & \text { for } n=2 m-1 \\ \left(2,1_{j-1}, 0_{m-j}\right) & \text { for } n=2 m .\end{cases}
$$

Because of the equation (27) and ellipticity of $\Delta_{j}$, the Hodge-de Rham decomposition holds on a compact manifold $(M, g)$,

$$
\Omega^{j}(M)=H^{j}(M) \oplus d\left(\Omega^{j-1}(M)\right) \oplus d^{*}\left(\Omega^{j+1}(M)\right),
$$

where $H^{j}(M)$ is the space of the harmonic $j$ forms. Therefore, if we know the eigenvalues of the Laplacian $\Delta_{j}$, then we have the eigenvalues of $d^{*} d, d d^{*}$. In addition, from the action of curvature $R_{\Lambda^{j}} / 2$, we can get the eigenvalues of $C^{*} C$. For example, on a space of constant curvature $K=c$, the curvature $R_{\Lambda^{j}} / 2$ acts by a constant $j(n-j) c$. We will calculate the eigenvalues of the operators on the standard sphere in the next section.

We move on to the higher spin Dirac operator on spinor fields coupled with differential forms. In other words, we shall study the case of the representation $\delta_{j}$ on $W_{\delta_{j}}$ with the highest weight

$$
\begin{cases}\left((3 / 2)_{j},(1 / 2)_{m-j}\right) \oplus\left((3 / 2)_{j},(1 / 2)_{m-j-1},-1 / 2\right) & \text { for } n=2 m \\ \left((3 / 2)_{j},(1 / 2)_{m-1-j}\right) & \text { for } n=2 m-1\end{cases}
$$

and the dimension $2^{[n / 2]} \frac{n-2 j+1}{n-j+1}\left(\begin{array}{c}n \\ j\end{array}\right)$, where $\delta_{j}$ splits into the sum of irreducible representation $\delta_{j}^{ \pm}$for $n=2 m$. These spinor fields are realized as sections of the tensor bundle $S_{0} \otimes \Lambda^{j}\left(T^{*} M^{c}\right)$, that is, spinor fields coupled with differential forms (see Remark 6.3). We consider the vector bundle with fiber $W_{\delta_{j}}$,

$$
E_{j}:=\operatorname{Spin}(M) \times_{\operatorname{Spin}(n)} W_{\delta_{j}} .
$$

There are four generalized gradients on $\Gamma\left(E_{j}\right)$ for $0 \leq j \leq[n / 2]$, 


$$
\begin{array}{lr}
U_{j}: \Gamma\left(E_{j}\right) \rightarrow \Gamma\left(E_{j, 1}\right) & \text { the (first) twistor operator, } \\
\widetilde{T}_{j}^{+}: \Gamma\left(E_{j}\right) \rightarrow \Gamma\left(E_{j+1}\right) & \text { the (second) twistor operator, } \\
\widetilde{D}_{j}: \Gamma\left(E_{j}\right) \rightarrow \Gamma\left(E_{j}\right) & \text { the higher spin Dirac opeator, } \\
\widetilde{T}_{j}^{-}: \Gamma\left(E_{j}\right) \rightarrow \Gamma\left(E_{j-1}\right) & \text { the co-twistor opeator, }
\end{array}
$$

where $E_{j, 1}$ is the vector bundle with the highest weight

$$
\begin{cases}\left(5 / 2,(3 / 2)_{j-1},(1 / 2)_{m-j}\right) \oplus\left(5 / 2,(3 / 2)_{j-1},(1 / 2)_{m-j-1},-1 / 2\right) & \text { for } n=2 m, \\ \left(5 / 2,(3 / 2)_{j-1},(1 / 2)_{m-1-j}\right) & \text { for } n=2 m-1 .\end{cases}
$$

Remark 6.1 When $j=0$, there are two generalized gradients on $E_{0}$,

$$
\widetilde{T}_{0}^{+}: \Gamma\left(E_{0}\right) \rightarrow \Gamma\left(E_{1}\right), \quad \widetilde{D}_{0}: \Gamma\left(E_{0}\right) \rightarrow \Gamma\left(E_{0}\right) .
$$

Then, we set $U_{0}=0$ and $\widetilde{T}_{0}^{-}=0$. When $n=2 m$ and $j=m$, there are only two generalized gradients on the bundle $E_{m}=E_{m}^{+} \oplus E_{m}^{-}$with the highest weight $\left((3 / 2)_{m}\right) \oplus\left((3 / 2)_{m-1},-3 / 2\right)$,

$$
U_{m}: \Gamma\left(E_{m}\right) \rightarrow \Gamma\left(E_{m, 1}\right), \quad \widetilde{T}_{m}^{-}: \Gamma\left(E_{m}\right) \rightarrow \Gamma\left(E_{m-1}\right) .
$$

Note that the higher spin Dirac operator does not exist. Then, we set $\widetilde{D}_{m}=0$ and $\widetilde{T}_{m}^{+}=0$ for $n=2 m$. When $n=2 m-1$ and $j=m-1$, there are three generalized gradients on the bundle $E_{m-1}$ with the highest weight $\left((3 / 2)_{m-1}\right)$,

$$
\begin{aligned}
& U_{m-1}: \Gamma\left(E_{m-1}\right) \rightarrow \Gamma\left(E_{m-1,1}\right), \quad \widetilde{T}_{m-1}^{-}: \Gamma\left(E_{m-1}\right) \rightarrow \Gamma\left(E_{m-2}\right), \\
& \widetilde{D}_{m-1}: \Gamma\left(E_{m-1}\right) \rightarrow \Gamma\left(E_{m-1}\right) .
\end{aligned}
$$

Then, we set $\widetilde{T}_{m-1}^{+}=0$ for $n=2 m-1$.

From now on, we assume that $(M, g)$ is a space of constant curvature $K=c$ with a spin structure. We calculate the Weitzenböck formulas on $\Gamma\left(E_{j}\right)$ from [16],

$$
\begin{aligned}
\Delta_{j}= & \nabla^{*} \nabla+\frac{1}{2} R_{\delta_{j}}=\nabla^{*} \nabla+\left(j(n-j+1)+\frac{n(n-1)}{8}\right) c \\
= & \frac{(n+2)(n-2 j)}{n-2 j+2} \widetilde{D}_{j}^{2}+\frac{4(n-2 j+1)(n-j+2)}{(n-2 j+3)(n-2 j+2)}\left(\widetilde{T}_{j}^{-}\right)^{*} \widetilde{T}_{j}^{-} \\
& +\left(j(n-j)-\frac{n(n-1)}{8}\right) c \\
= & -\frac{4(n-2 j+1)(j+1)}{(n-2 j-1)(n-2 j)}\left(\widetilde{T}_{j}^{+}\right)^{*} \widetilde{T}_{j}^{+}+\frac{(n-2 j+2)(n+2)}{n-2 j} \widetilde{D}_{j}^{2} \\
& \quad+\left((j-1)(n-j+1)-\frac{n(n-1)}{8}\right) c \\
= & \frac{(n-j+2)(n+2)}{(n-j+1)(n+1)} U_{j}^{*} U_{j}+\frac{(n-2 j)(n-2 j+1)}{(n-2 j-1)(n-j+1)}\left(\widetilde{T}_{j}^{+}\right)^{*} \widetilde{T}_{j}^{+} \\
& \quad+\left(j(n-j+2)+\frac{n(n+1)}{8}\right) c .
\end{aligned}
$$


If denominators in the above equation are zero, then we ignore the equation. For example, when $n=2 m$ and $j=m$, we do not consider the third equality. From formulas in [17] and the equation (18) in Lemma 4.2, we also have

$$
\begin{aligned}
& \frac{1}{\sqrt{n-2 j}} \widetilde{D}_{j} \widetilde{T}_{j-1}^{+}=\frac{1}{\sqrt{n-2 j+4}} \widetilde{T}_{j-1}^{+} \widetilde{D}_{j-1}, \quad \widetilde{T}_{j+1}^{+} \widetilde{T}_{j}^{+}=0, \quad \widetilde{T}_{j-1}^{-} \widetilde{T}_{j}^{-}=0, \\
& \left(\widetilde{T}_{j-1}^{+}\right)^{*} \widetilde{T}_{j-1}^{+}=\frac{(n-2 j+1)(n-j+2)}{j(n-2 j+3)} \widetilde{T}_{j}^{-}\left(\widetilde{T}_{j}^{-}\right)^{*} .
\end{aligned}
$$

We normalize these generalized gradients

$$
\begin{aligned}
& D_{j}:=\sqrt{\frac{(n+2)(n-2 j)}{n-2 j+2}} \widetilde{D}_{j}, \\
& T_{j}^{-}:=2 \sqrt{\frac{(n-2 j+1)(n-j+2)}{(n-2 j+3)(n-2 j+2)}} \widetilde{T}_{j}^{-}, \quad T_{j}^{+}:=2 \sqrt{\frac{j+1}{n-2 j}} \widetilde{T}_{j}^{+} .
\end{aligned}
$$

to compare with other articles as Remark 6.3. We summarize the calculations so far in the next theorem.

Theorem 6.2 Let $(M, g)$ be a space of constant curvature $K=c$ with a spin structure. The generalized gradients on $\Gamma\left(E_{j}\right)$ satisfy

$$
\begin{aligned}
\Delta_{j}= & D_{j}^{2}+\left(T_{j}^{-}\right)^{*} T_{j}^{-}+\left(j(n-j)-\frac{n(n-1)}{8}\right) c \\
= & -\frac{n-2 j+1}{n-2 j-1}\left(T_{j}^{+}\right)^{*} T_{j}^{+}+\frac{(n-2 j+2)^{2}}{(n-2 j)^{2}} D_{j}^{2}+\left((j-1)(n-j+1)-\frac{n(n-1)}{8}\right) c, \\
& D_{j} T_{j-1}^{+}=\frac{n-2 j}{n-2 j+2} T_{j-1}^{+} D_{j-1}, \quad T_{j+1}^{+} T_{j}^{+}=0, \quad T_{j-1}^{-} T_{j}^{-}=0, \quad T_{j-1}^{+}=\left(T_{j}^{-}\right)^{*} .
\end{aligned}
$$

Remark 6.3 In [8], these operators are realized as components of the twisted Dirac operator $D(j)$ on $S_{0} \otimes \Lambda^{j}\left(T^{*} M^{c}\right)$ up to a constant multiple. Indeed, the set of the highest weights of irreducible summands in $S_{0} \otimes \Lambda^{j}\left(T^{*} M^{c}\right)$ is given by

$$
\left\{\lambda=\left(1_{j}, 0_{m-j}\right)+\left(\epsilon_{1} / 2, \cdots, \epsilon_{m} / 2\right) \mid \lambda \text { satisfies the dominant condition, } \epsilon_{i} \in\{ \pm 1\}\right\}
$$

because each nonzero weight $\left(\epsilon_{1} / 2, \cdots, \epsilon_{m} / 2\right)$ of the spinor representation has multiplicity one. Then, we decompose the tensor bundle into the sum of irreducible bundles,

$$
S_{0} \otimes \Lambda^{j}\left(T^{*} M^{c}\right)=E_{j} \oplus E_{j-1} \oplus \cdots \oplus E_{0} .
$$

Along this bundle decomposition, the twisted Dirac operator $D(j)$ is represented as

$$
D(j)=\left(\begin{array}{cccccc}
D(j)_{j} & T(j)_{j-1}^{+} & 0 & 0 & \ldots & 0 \\
T(j)_{j}^{-} & D(j)_{j-1} & T(j)_{j-2}^{+} & 0 & \ldots & 0 \\
0 & T(j)_{j-1}^{-} & D(j)_{j-2} & T(j)_{j-3} & \ddots & 0 \\
\vdots & \ddots & \ddots & \ddots & \ddots & \vdots \\
0 & 0 & 0 & \ldots & D(j)_{1} & T(j)_{0}^{+} \\
0 & 0 & 0 & \ldots & T(j)_{1}^{-} & D(j)_{0}
\end{array}\right)
$$


where $D(j)_{k}$ (resp. $T(j)_{k}^{ \pm}$) is a nonzero constant multiple of $\widetilde{D}_{k}: \Gamma\left(E_{k}\right) \rightarrow \Gamma\left(E_{k}\right)$ (resp. $\left.\widetilde{T}_{k}^{ \pm}: \Gamma\left(E_{k}\right) \rightarrow \Gamma\left(E_{k \pm 1}\right)\right)$ for $k=0, \ldots, j$. Considering the square of $D(j)$, we have Weitzenböck formulas in Theorem 6.2 and check that $D(j)_{j}, T(j)_{j}^{-}$and $T(j)_{j-1}^{+}$coincide with our $D_{j}$, $T_{j}^{-}$and $T_{j-1}^{+}$, respectively.

As applications of Theorem 6.2, we obtain a factorization formula and the Hodge-de Rham decomposition with respect to the operators on $\Gamma\left(E_{j}\right)$.

Corollary 6.4 (Factorization formula) We consider a space $(M, g)$ of constant curvature $K=c$ with a spin structure. On $\Gamma\left(E_{j}\right)$ for $1 \leq j \leq[n / 2]-1$, we have

$$
\begin{aligned}
0=\left(D_{j}^{2}-\frac{(n-2 j)^{2}}{(n-2 j+2)^{2}}\right. & \left.\left(\Delta_{j}-\left((j-1)(n-j+1)-\frac{n(n-1)}{8}\right) c\right)\right) \\
& \times\left(D_{j}^{2}-\left(\Delta_{j}-\left(j(n-j)-\frac{n(n-1)}{8}\right) c\right)\right) .
\end{aligned}
$$

Proof The corollary follows from $\left(T_{j}^{+}\right)^{*} T_{j}^{+}\left(T_{j}^{-}\right)^{*} T_{j}^{-}=\left(T_{j}^{+}\right)^{*} T_{j}^{+} T_{j-1}^{+} T_{j}^{-}=0$.

Eliminating $D_{j}^{2}$ from two equations for $\Delta_{j}$ in Theorem 6.2, we get

$$
\Delta_{j}=\frac{(n-2 j)^{2}}{4(n-2 j-1)}\left(T_{j}^{+}\right)^{*} T_{j}^{+}+\frac{(n-2 j+2)^{2}}{4(n-2 j+1)}\left(T_{j}^{-}\right)^{*} T_{j}^{-}+\frac{n(n+1)}{8} c .
$$

Taking the usual Hodge-de Rham decomposition as a model, we can show the following $L^{2}$-orthogonal decomposition.

Proposition 6.5 On a compact spin manifold $(M, g)$ of constant sectional curvature $K=c$, we have the Hodge-de Rham decomposition for spinor fields coupled with differential forms,

$$
\begin{aligned}
\Gamma\left(E_{j}\right) & =T_{j-1}^{+}\left(\Gamma\left(E_{j-1}\right)\right) \oplus T_{j+1}^{-}\left(\Gamma\left(E_{j+1}\right)\right) \oplus \operatorname{ker}\left(\Delta_{j}-\frac{n(n+1)}{8} c\right), \\
\operatorname{ker} T_{j}^{+} & =T_{j-1}^{+}\left(\Gamma\left(E_{j-1}\right)\right) \oplus \operatorname{ker}\left(\Delta_{j}-\frac{n(n+1)}{8} c\right), \\
\operatorname{ker} T_{j}^{-} & =T_{j+1}^{-}\left(\Gamma\left(E_{j-1}\right)\right) \oplus \operatorname{ker}\left(\Delta_{j}-\frac{n(n+1)}{8} c\right) .
\end{aligned}
$$

Here, we exclude the case of $n=2 m-1$ and $j=m-1$. When $j=0$, the first equation means

$$
\Gamma\left(E_{0}\right)=T_{1}^{-}\left(\Gamma\left(E_{1}\right)\right) \oplus \operatorname{ker}\left(\Delta_{0}-\frac{n(n+1)}{8} c\right) .
$$

When $n=2 m$ and $j=m$, it does

$$
\Gamma\left(E_{m}\right)=T_{m-1}^{+}\left(\Gamma\left(E_{m-1}\right)\right) \oplus \operatorname{ker}\left(\Delta_{m}-\frac{n(n+1)}{8} c\right) .
$$

Note that $\operatorname{ker}\left(\Delta_{j}-\frac{n(n+1)}{8} c\right)$ is zero on a compact spin manifold of positive constant curvature for $j \geq 1$ because we have $\Delta_{j}-\frac{n(n+1)}{8} c \geq j(n-j+2) c$ from the last equality in (29). 


\section{Harmonic analysis for spinor fields with differential forms on $S^{n}$}

We review a well-known result for the eigenvalues of the operators $\left\{\Delta_{j}, d d^{*}, d^{*} d, C^{*} C\right\}$ acting on differential forms on the standard sphere, $[13,20]$. Let $V_{j}(k)$ be an irreducible $\mathrm{SO}(n+1)$-module with the highest weight

$$
\left(k+1,1_{j-1}, 0_{m-j}\right)=\left(k, 0_{m-1}\right)+\left(1_{j}, 0_{m-j}\right)
$$

for $0 \leq j \leq m=\left[\frac{n+1}{2}\right]$. Then, Frobenius reciprocity and the branching rule give us the decomposition of $L^{2}\left(S^{n}, \Lambda^{j}\left(T^{*} M^{c}\right)\right)$ as an $\mathrm{SO}(n+1)$-module.

1. For $j=0$,

$$
L^{2}\left(S^{n}, \Lambda^{0}\left(T^{*} M^{c}\right)\right) \cong \bigoplus_{k \geq 0} V_{1}(k) \oplus V_{0}(0), \quad \operatorname{ker} d=H^{0}\left(S^{n}\right) \cong V_{0}(0) .
$$

2. For $n=2 m$ and $j=m$,

$$
L^{2}\left(S^{2 m}, \Lambda^{m}\left(T^{*} M^{c}\right)\right) \cong \bigoplus_{k \geq 0} 2 V_{m}(k), \quad \operatorname{ker} d^{*} \cong \bigoplus_{k \geq 0} V_{m}(k), \quad \operatorname{ker} d \cong \bigoplus_{k \geq 0} V_{m}(k) .
$$

3. For $n=2 m-1$ and $j=m-1$,

$$
\begin{gathered}
L^{2}\left(S^{2 m-1}, \Lambda^{m-1}\left(T^{*} M^{c}\right)\right) \cong \bigoplus_{k \geq 0} V_{m}(k) \oplus \bigoplus_{k \geq 0} V_{m-1}(k), \\
V_{m}(k):=V_{m}^{+}(k) \oplus V_{m}^{-}(k), \quad \operatorname{ker} d^{*} \cong \bigoplus_{k \geq 0} V_{m}(k), \quad \operatorname{ker} d \cong \bigoplus_{k \geq 0} V_{m-1}(k),
\end{gathered}
$$

where $V_{m}^{ \pm}(k)$ is an irreducible $\mathrm{SO}(2 m)$-module with the highest weight $\left(k+1,1_{m-2}, \pm 1\right)$

4. Otherwise,

$$
\begin{array}{r}
L^{2}\left(S^{n}, \Lambda^{j}\left(T^{*} M^{c}\right)\right) \cong \bigoplus_{k \geq 0} V_{j+1}(k) \oplus \bigoplus_{k \geq 0} V_{j}(k), \\
\operatorname{ker} d^{*} \cong \bigoplus_{k \geq 0} V_{j+1}(k), \quad \text { ker } d \cong \bigoplus_{k \geq 0} V_{j}(k) .
\end{array}
$$

We calculate the eigenvalue of $\Delta_{j}$ on $V_{j}(k)$ by Freudenthal's formula. Then, the next proposition follows from (27) and (28).

Proposition 7.1 ([13, 20]) The eigenvalues of the operators $\left\{\Delta_{j}, d d^{*}, d^{*} d, C^{*} C\right\}$ on $V_{j+1}(k)$ and $V_{j}(k)$ are given as follows:

\begin{tabular}{|c|c|c|}
\hline & on $V_{j+1}(k) \subset$ ker $d^{*}$ & on $V_{j}(k) \subset \operatorname{ker} d$ \\
\hline$\Delta_{j}$ & $(k+j+1)(n+k-j)$ & $(k+j)(n-j+k+1)$ \\
\hline$d d^{*}$ & 0 & $(k+j)(n-j+k+1)$ \\
\hline$d^{*} d$ & $(k+j+1)(n+k-j)$ & 0 \\
\hline$C^{*} C$ & $\frac{j}{j+1} k(n+k+1)$ & $\frac{n-j}{n-j+1} k(n+k+1)$ \\
\hline
\end{tabular}

In particular, the space of the Killing $j$-forms $\operatorname{ker} C \cap \operatorname{ker} d^{*}$ is isomorphic to $V_{j+1}(0)$, and the space of the co-Killing $j$-forms $\operatorname{ker} C \cap \operatorname{ker} d$ is isomorphic to $V_{j}(0)$. 
The (co-) Killing forms have interesting geometric meaning like Killing tensor fields [24].

We shall study harmonic analysis for the spinor fields coupled with differential forms on the standard sphere. The space $L^{2}\left(S^{n}, E_{j}\right)$ is decomposed as $\operatorname{Spin}(n+1)$-module.

Proposition 7.21 . In the case of $n=2 m$, we denote by $V_{j}(k)^{\prime}$ an irreducible $\operatorname{Spin}(2 m+1)$ -module with the highest weight

$$
\left(k+3 / 2,(3 / 2)_{j-1},(1 / 2)_{m-j}\right)=\left(k+1 / 2,(1 / 2)_{m-1}\right)+\left(1_{j}, 0_{m-j}\right)
$$

for $k=0,1,2, \ldots$. Then,

1. $\operatorname{For} j=0$,

$$
\begin{aligned}
& L^{2}\left(S^{2 m}, E_{0}^{ \pm}\right) \cong \bigoplus_{k \geq 0} V_{1}(k)^{\prime} \oplus V_{0}(0)^{\prime}, \quad L^{2}\left(S^{2 m}, E_{0}\right) \cong \bigoplus_{k \geq 0} 2 V_{1}(k)^{\prime} \oplus 2 V_{0}(0)^{\prime}, \\
& \operatorname{ker} T_{0}^{+} \cong 2 V_{0}(0)^{\prime} .
\end{aligned}
$$

2. For $1 \leq j \leq m-1$,

$$
\begin{aligned}
& L^{2}\left(S^{2 m}, E_{j}^{ \pm}\right) \cong \bigoplus_{k \geq 0} V_{j+1}(k)^{\prime} \oplus \bigoplus_{k \geq 0} V_{j}(k)^{\prime}, \\
& L^{2}\left(S^{2 m}, E_{j}\right) \cong \bigoplus_{k \geq 0} 2 V_{j+1}(k)^{\prime} \oplus \bigoplus_{k \geq 0} 2 V_{j}(k)^{\prime}, \\
& \operatorname{ker} T_{j}^{-} \cong \bigoplus_{k \geq 0} 2 V_{j+1}(k)^{\prime}, \quad \operatorname{ker} T_{j}^{+} \cong \bigoplus_{k \geq 0} 2 V_{j}(k)^{\prime} .
\end{aligned}
$$

3. For $j=m$,

$$
L^{2}\left(S^{2 m}, E_{m}^{ \pm}\right) \cong \bigoplus_{k \geq 0} V_{m}(k)^{\prime}, \quad L^{2}\left(S^{2 m}, E_{j}\right) \cong \bigoplus_{k \geq 0} 2 V_{m}(k)^{\prime}, \quad \operatorname{ker} T_{m}^{-}=\{0\} .
$$

In the case of $n=2 m-1$, we denote by $V_{j}^{ \pm}(k)^{\prime}$ an irreducible $\operatorname{Spin}(2 m)$-module with the highest weight

$$
\left(k+3 / 2,(3 / 2)_{j-1},(1 / 2)_{m-j-1}, \pm 1 / 2\right)=\left(k+1 / 2,(1 / 2)_{m-1}, \pm 1 / 2\right)+\left(1_{j}, 0_{m-j}\right)
$$

for $k=0,1,2, \ldots$, and put $V_{j}(k)^{\prime}=V_{j}^{+}(k) \oplus V_{j}^{-}(k)$. Then,

1. $\operatorname{For} j=0$,

$$
L^{2}\left(S^{2 m-1}, E_{0}\right) \cong \bigoplus_{k \geq 0} V_{1}(k)^{\prime} \oplus V_{0}(0)^{\prime}, \quad \operatorname{ker} T_{0}^{+} \cong V_{0}(0)^{\prime} .
$$

2. For $1 \leq j \leq m-2$,

$$
\begin{array}{r}
L^{2}\left(S^{2 m-1}, E_{j}\right) \cong \bigoplus_{k \geq 0} V_{j+1}(k)^{\prime} \oplus \bigoplus_{k \geq 0} V_{j}(k)^{\prime}, \\
\operatorname{ker} T_{j}^{-} \cong \bigoplus_{k \geq 0} V_{j+1}(k)^{\prime}, \quad \operatorname{ker} T_{j}^{+} \cong \bigoplus_{k \geq 0} V_{j}(k)^{\prime} .
\end{array}
$$


3. For $j=m-1$,

$$
L^{2}\left(S^{2 m-1}, E_{m-1}\right) \cong \bigoplus_{k \geq 0} V_{m}(k)^{\prime} \oplus \bigoplus_{k \geq 0} V_{m-1}(k)^{\prime}, \quad \operatorname{ker} T_{m-1}^{-} \cong \bigoplus_{k \geq 0} V_{m}(k)^{\prime}
$$

We know already how the sections of $\left\{E_{j}\right\}_{j}$ relate to each other through operators $\left\{T_{j}^{ \pm}\right\}_{j}$. In fact, $\operatorname{ker} T_{j}^{+}=$Image $T_{j-1}^{+}$and $\operatorname{ker} T_{j}^{-}=$Image $T_{j+1}^{-}$. Then, all we have to do is to calculate the eigenvalues of the operators on $\Gamma\left(E_{j}\right)$. The eigenvalues of $D_{j}^{2}$ were calculated in [8] by using a method from parabolic geometry in [7]. We calculate them only from the eigenvalues of $\Delta_{j}$ and Weitzenböck formulas.

Theorem 7.3 The eigenvalues of $D_{j}^{2},\left(T_{j}^{-}\right)^{*} T_{j}^{-},\left(T_{j}^{+}\right)^{*} T_{j}^{+}$and $U_{j}^{*} U_{j}$ on $L^{2}\left(S^{n}, E_{j}\right)$ are given as follows:

\begin{tabular}{|c|c|c|}
\hline & on $V_{j+1}(k)^{\prime} \subset \operatorname{ker} T_{j}^{-}$ & on $V_{j}(k)^{\prime} \subset \operatorname{ker} T_{j}^{+}$ \\
\hline$\Delta_{j}$ & $(k+j+1)(n-j+k+1)+\frac{n(n+1)}{8}$ & $(k+j)(n-j+k+2)+\frac{n(n+1)}{8}$ \\
\hline$D_{j}^{2}$ & $\left(\frac{n}{2}+k+1\right)^{2}$ & $\frac{(n-2 j)^{2}}{(n-2 j+2)^{2}}\left(\frac{n}{2}+k+1\right)^{2}$ \\
\hline$\left(T_{j}^{-}\right)^{*} T_{j}^{-}$ & 0 & $\frac{4(n-2 j+1)}{(n-2 j+2)^{2}}(k+j)(n-j+k+2)$ \\
\hline$\left(T_{j}^{+}\right)^{*} T_{j}^{+}$ & $\frac{4(n-2 j-1)}{(n-2 j)^{2}}(n-j+k+1)(k+j+1)$ & 0 \\
\hline$U_{j}^{*} U_{j}$ & $\frac{(n+1) j}{(n+2)(j+1)} k(n+k+2)$ & $\frac{(n+1)(n-j+1)}{(n+2)(n-j+2)} k(n+k+2)$ \\
\hline
\end{tabular}

In particular, for $n=2 m-1($ resp. $n=2 m)$, $\operatorname{ker} U_{j} \cap \operatorname{ker} T_{j}^{-} \cong V_{j+1}(0)^{\prime}\left(\right.$ resp. $\left.2 V_{j+1}(0)^{\prime}\right)$ and $\operatorname{ker} U_{j} \cap \operatorname{ker} T_{j}^{+} \cong V_{j}(0)^{\prime}\left(\operatorname{resp} .2 V_{j}(0)^{\prime}\right)$.

\section{A Appendix: calculation}

In this appendix, we give the calculation of the eigenvalues of Casimir elements and Weitzenböck formulas needed in Sect. 2. For more detail, see [16]. Let $\left(\pi_{j}, W_{j}\right)$ be an irreducible representation with the highest weight $\left(j+1 / 2,(1 / 2)_{[n / 2]-1}\right)$. The conformal weights $\{w(\lambda)\}_{\lambda}$ and the shifted conformal weights $\left\{\widehat{w}(\lambda)=w(\lambda)+\frac{n-1}{2}\right\}_{\lambda}$ associated with the irreducible summand of $W_{j} \otimes \mathbb{C}^{n}$ are given by

$$
\begin{aligned}
w\left(\pi_{j+1}\right) & :=w\left(\pi_{j+1} ; \pi_{j}\right)=j+1 / 2, \quad \widehat{w}\left(\pi_{j+1}\right)=j+n / 2, \\
w\left(\pi_{j, 1}\right) & :=w\left(\pi_{j, 1} ; \pi_{j}\right)=-1 / 2, \quad \widehat{w}\left(\pi_{j, 1}\right)=n / 2-1, \\
w\left(\pi_{j}\right) & :=w\left(\pi_{j} ; \pi_{j}\right)=-n / 2+1 / 2, \quad \widehat{w}\left(\pi_{j}\right)=0, \\
w\left(\pi_{j-1}\right) & :=w\left(\pi_{j-1} ; \pi_{j}\right)=-n-j+3 / 2, \quad \widehat{w}\left(\pi_{j-1}\right)=1-j-n / 2 .
\end{aligned}
$$

Then, we can easily calculate the relative dimensions,

$$
\begin{aligned}
& \frac{\operatorname{dim} W_{j+1}}{\operatorname{dim} W_{j}}=\frac{n+j-1}{j+1}, \quad \frac{\operatorname{dim} W_{j, 1}}{\operatorname{dim} W_{j}}=\frac{(n-3)(n+j-1) j}{(n+j-2)(j+1)}, \\
& \frac{\operatorname{dim} W_{j}}{\operatorname{dim} W_{j}}=1, \quad \frac{\operatorname{dim} W_{j-1}}{\operatorname{dim} W_{j}}=\frac{j}{n+j-2} .
\end{aligned}
$$


The Casimir element $c_{k}$ with order $k$ is an $\mathrm{SO}(n)$-invariant element in the enveloping algebra $U(\mathfrak{g} \mathfrak{o}(n))$ given by

$$
c_{k}=\sum_{1 \leq i_{1}, i_{2} \ldots i_{k} \leq n} e_{i_{1} i_{2}} e_{i_{2} i_{3}} \ldots e_{i_{k} i_{1}},
$$

where $\left\{e_{i j}=e_{i} \wedge e_{j}\right\}_{i<j}$ is a standard basis for $\mathfrak{g} \mathfrak{o}(n)$. Shifting $e_{i j}$ to $\hat{e}_{i j}=e_{i j}+\frac{n-1}{2}$, we define the shifted Casimir element $\widehat{c}_{k}=\sum \widehat{e}_{i_{1} i_{2}} \widehat{e}_{i_{2} i_{3}} \ldots \widehat{e}_{i_{k} i_{1}}$. The eigenvalue of $c_{k}$ on $W_{j}^{2}$ is computed by the conformal weights and the relative dimensions.

$$
\pi_{j}\left(c_{k}\right)=\sum_{\lambda} w(\lambda)^{k} \frac{\operatorname{dim} W_{\lambda}}{\operatorname{dim} W_{j}}, \quad \pi_{j}\left(\widehat{c}_{k}\right)=\sum_{\lambda} \widehat{w}(\lambda)^{k} \frac{\operatorname{dim} W_{\lambda}}{\operatorname{dim} W_{j}},
$$

where $\lambda$ runs among $\pi_{j+1}, \pi_{j, 1}, \pi_{j}$ and $\pi_{j-1}$. Since the number of the generalized gradients on $S_{j}$ is four, there are two independent Weitzenböck formulas. In our case, we have the following two independent formulas on $S_{j}$. One is

$$
-\frac{1}{2} R_{j}^{1}=w\left(\pi_{j+1}\right)\left(\widetilde{T}_{j}^{+}\right)^{*} \widetilde{T}_{j}^{+}+w\left(\pi_{j, 1}\right) U_{j}^{*} U_{j}+w\left(\pi_{j}\right) \widetilde{D}_{j}^{2}+w\left(\pi_{j-1}\right)\left(\widetilde{T}_{j}^{-}\right)^{*} \widetilde{T}_{j}^{-} .
$$

The second one is complicated,

$$
\widehat{R}_{j}^{4}=a\left(\pi_{j+1}\right)\left(\widetilde{T}_{j}^{+}\right)^{*} \widetilde{T}_{j}^{+}+a\left(\pi_{j, 1}\right) U_{j}^{*} U_{j}+a\left(\pi_{j}\right) \widetilde{D}_{j}^{2}+a\left(\pi_{j-1}\right)\left(\widetilde{T}_{j}^{-}\right)^{*} \widetilde{T}_{j}^{-},
$$

where

$$
a(\lambda)=\sum_{p=0}^{3} \pi_{j}\left(\widehat{c}_{3-p}\right)(-\widehat{w}(\lambda))^{p}, \quad \text { for } \lambda=\pi_{j+1}, \pi_{j, 1}, \pi_{j}, \pi_{j-1} .
$$

Furthermore, the curvature actions $R_{j}^{1}$ and $\widehat{R}_{j}^{4}$ on the standard sphere are

$$
R_{j}^{1}=\pi_{j}\left(c_{2}\right), \quad \widehat{R}_{j}^{4}=\pi_{j}\left(\widehat{c}_{5}-\frac{n-1}{2} \widehat{c}_{4}\right) .
$$

On the other hand, it follows form the definition of the generalized gradients that

$$
\nabla^{*} \nabla=\left(\widetilde{T}_{j}^{+}\right)^{*} \widetilde{T}_{j}^{+}+U_{j}^{*} U_{j}+\widetilde{D}_{j}^{2}+\left(\widetilde{T}_{j}^{-}\right)^{*} \widetilde{T}_{j}^{-} .
$$

Deleting two generalized gradients from the above equation by using two Weitzenböck formulas, we obtain (9) and (10).

Acknowledgements This work was partially supported by JSPS KAKENHI (Grant Number JP19K03480) and Waseda University Grant for Special Research Projects (Project number: 2020C-614). We thank the anonymous referee for helpful comments.

Open Access This article is licensed under a Creative Commons Attribution 4.0 International License, which permits use, sharing, adaptation, distribution and reproduction in any medium or format, as long as you give appropriate credit to the original author(s) and the source, provide a link to the Creative Commons licence, and indicate if changes were made. The images or other third party material in this article are included in the article's Creative Commons licence, unless indicated otherwise in a credit line to the material. If material is not included in the article's Creative Commons licence and your intended use is not permitted by statutory regulation or exceeds the permitted use, you will need to obtain permission directly from the copyright holder. To view a copy of this licence, visit http://creativecommons.org/licenses/by/4.0/. 


\section{References}

1. Bär, C., Mazzeo, R. Manifolds with many Rarita-Schwinger fields, Comm. Math. Phys. 384(1), 533-548 (2021)

2. Besse, A.: Einstein manifolds. Springer, Berlin (1987)

3. Branson, T.: Harmonic analysis in vector bundles associated to the rotation and spin groups. J. Funct. Anal. 106, 314-328 (1992)

4. Branson, T.: Stein-Weiss operators and ellipticity. J. Funct. Anal. 151, 334-383 (1997)

5. Branson, T.: Second order conformal covariants. Proc. Amer. Math. Soc. 126(4), 1031-1042 (1998)

6. Branson, T.: Spectra of self-gradients on spheres. J. Lie Theory 9(2), 491-506 (1999)

7. Branson, T., Ólafsson, G., Ørsted, B.: Spectrum generating operators and intertwining operators for representations induced from a maximal parabolic subgroup. J. Funct. Anal. 135, 163-205 (1996)

8. Bureš, J., Souček, V.: Eigenvalues of conformally invariant operators on spheres, in "Proc. Winter School, 1998”, Suppl. Rend. Circ. Mat. Palermo, II 59, 109-122 (1999)

9. Bureš, J., Sommen, F., Souček, V., Van Lancker, P.: Rarita-Schwinger type operators in Clifford analysis. J. Funct. Anal. 185(2), 425-455 (2001)

10. Bureš, J., Sommen, F., Souček, V., Van Lancker, P.: Symmetric analogues of Rarita-Schwinger equations. Ann. Global Anal. Geom. 21(3), 215-240 (2002)

11. Eelbode, D., Šmid, D.: Factorization of laplace operators on higher spin representations. Complex anal. Oper. Theory 6(5), 1011-1023 (2012)

12. Fegan, H.D.: Conformally invariant first order differential operators. Quart. J. Math. Oxford 27, 371378 (1976)

13. Folland, G.B.: Harmonic analysis of the de Rham complex on the sphere. J. Reine Angew. Math. 398, 130-143 (1989)

14. Heil, K., Moroianu, A., Semmelmann, U.: Killing and conformal Killing tensors. J. Geom. Phys. 106, 383-400 (2016)

15. Herzlich, M.: Universal positive mass theorems. Comm. Math. Phys. 351, 973-992 (2017)

16. Homma, Y.: Bochner-Weitzenböck formula and curvature actions on Riemannian manifolds. Trans. Amer. Math. Soc. 358, 87-114 (2006)

17. Homma, Y.: Twisted Dirac operators and generalized gradients. Ann. Global Anal. Geom. 50(2), 101127 (2016)

18. Homma, Y., Semmelmann, U.: The kernel of the Rarita-Schwinger operator on Riemannian spin manifolds. Comm. Math. Phys. 370(3), 853-871 (2019)

19. Homma, Y., Tomihisa, T.: Spectra of the Rarita-Schwinger operators on some symmetric spaces. J. Lie Theory 31(1), 249-264 (2021)

20. Ikeda, A., Taniguchi, Y.: Spectra and eigenforms of the Laplacian on $S^{n}$ and $P^{n}(\mathbb{C})$. Osaka J. Math. 15(3), 515-546 (1978)

21. Kalina, J., Ørsted, B., Pierzchalski, A., Walczak, P., Zang, G.: Elliptic gradients and highest weights. Bull. Polon. Acad. Sci. Ser. Math. 44, 511-519 (1996)

22. Pilca, M.: A new proof of Branson's classification of elliptic generalized gradients. Manuscripta Math. 136, 65-81 (2011)

23. Semmelmann, U., Weingart, G.: An upper bound for a Hilbert polynomial on quaternionic Kähler manifolds. J. Geom. Anal. 14, 151-170 (2004)

24. Semmelmann, U.: Conformal killing forms on Riemannian manifolds. Math. Z. 245, 503-527 (2003)

25. Sumitomo T., Tandai K.: Killing tensor fields on the standard sphere and spectra of $\mathrm{SO}(n+1) /(\mathrm{SO}(n-1) \times \mathrm{SO}(2))$ and $\mathrm{O}(n+1) /(O(n-1) \times \mathrm{O}(2))$, Osaka J. Math., 20, 51-78 (1983). https://doi.org/10.18910/8429

26. Takeuchi, M.: Killing tensor fields on spaces of constant curvature. Tsukuba J. Math. 7, 233-255 (1983)

27. Želobenko, D.P.: Compact lie groups and their representations, Transl, Math. Mono., Vol.40, AMS (1973)

Publisher's Note Springer Nature remains neutral with regard to jurisdictional claims in published maps and institutional affiliations. 